
7

\title{
Demography and linked selection interact to shape the genomic landscape of codistributed woodpeckers during the Ice Age
}

Short title: Demography and selection shape avian genomic landscape

Lucas R. Moreira ${ }^{1,2,3^{*}}$, John Klicka ${ }^{4}$, Brian Tilston Smith ${ }^{3}$

${ }^{1}$ Department of Ecology, Evolution, and Environmental Biology, Columbia University, New York, NY, USA.

${ }^{2}$ Department of Ornithology, American Museum of Natural History, New York City, NY, USA.

${ }^{3}$ Program in Bioinformatics and Integrative Biology, University of Massachusetts Chan Medical School, Worcester, MA, USA.

${ }^{4}$ Burke Museum of Natural History and Culture and Department of Biology, University of Washington, Seattle, WA, USA.

* corresponding author, 1r2767@ columbia.edu

\begin{abstract}
The glacial cycles of the Pleistocene had a global impact on the evolution of species. Although the influence of genetic drift on population genetic dynamics is well understood, the role of selection in shaping patterns of genomic variation during these dramatic climatic changes is less clear. We used whole genome resequencing data to investigate the interplay between demography and natural selection and their influence on the genomic landscape of Downy and Hairy Woodpecker, species co-distributed in previously glaciated North America. Our results revealed a dynamic population history with repeated cycles of bottleneck and expansion, and genetic structure associated with glacial refugia. Levels of nucleotide diversity varied substantially along the genomes of Downy and Hairy Woodpecker, but this variation was highly correlated between the two species, suggesting the presence of conserved genomic features. Nucleotide diversity in both species was positively correlated with recombination rate and negatively correlated with gene density, suggesting that linked selection played a role in reducing diversity in regions of low recombination and high density of targets of selection. Despite strong temporal fluctuations in $\mathrm{N}_{\mathrm{e}}$, our demographic analyses indicate that Downy and Hairy Woodpecker were able to maintain relatively large effective population sizes during glaciations, which might have favored natural selection. The magnitude of the effect of linked selection seems to have been modulated by the individual demographic trajectory of populations and species, such that purifying selection has been more efficient in removing deleterious alleles in Hairy Woodpecker owing to its larger long-term $\mathrm{N}_{\mathrm{e}}$. These results highlight that while drift captures the expected signature of contracting and expanding populations during climatic perturbations, the interaction of multiple processes produces a predictable and highly heterogeneous genomic landscape.
\end{abstract}




\section{Introduction}

Pleistocene glacial cycles altered the distribution and evolution of entire communities [1,2]. Despite the profound impact glaciations had on the evolutionary trajectory of species, the majority of research on the topic has focused on how demographic dynamics have shaped neutral genetic variation [2,3]. Population expansion [4,5], genetic structuring in refugia [6-10], and decreased diversity in expanding populations [11-13] are among the most common patterns recovered. However, as species rapidly expanded and colonized areas under extreme environmental change they would have been subject to strong selective pressures, such as increased tolerance to cold and selection against deleterious mutations [14,15]. Understanding how natural selection, along with genetic drift, interact with features of the genome to shape the genomic landscape of diversity and differentiation will clarify the broader significance of the Ice Age on the evolution of species.

Demography and natural selection play a central role shaping levels of genetic diversity, but their effects are intertwined [16-18]. Neutral genetic diversity $(\theta)$ is the product of the rate at which new alleles are generated (i.e., mutation rate $\mu$ ) by the effective population size $\left(\mathrm{N}_{\mathrm{e}}\right)$, so that diversity levels are predicted to increase as a function of the size of populations (In diploids, $\theta=4 \mathrm{~N}_{\mathrm{e}} \mu[19,20]$ ). On the other hand, fixation of beneficial alleles (selective sweep [21,22]) or removal of deleterious mutations (background selection [22-25]) can cause genetic diversity to decrease across the genome through the effect of linked selection [24]. Demographic perturbations that cause $\mathrm{N}_{\mathrm{e}}$ to fluctuate over time and space (e.g., glacial bottlenecks) are, therefore, expected to result in a larger accumulation of mildly deleterious alleles when compared to large populations with constant $\mathrm{N}_{\mathrm{e}}$ because of the reduced efficacy of purifying selection when genetic drift is strong [26-30]. Hence, populations resulting from founder events, such as at the leading edge of a postglacial expansion, often show elevated genetic load [27,30,31].

Levels of diversity and differentiation along the genome also vary due to the differing effects of intrinsic genomic properties [32-36]. Genome features such as variation in mutation rate, recombination rate, distribution of functional elements, and nucleotide composition impact the rates at which genetic variants are produced, maintained, and lost [37]. Regions enriched for functional elements (e.g., coding sequences), for instance, tend to exhibit significantly lower levels of genetic diversity due to the recurrent effect of natural selection [33,38-40]. The loss of variation is further amplified by linkage disequilibrium (LD), which reduces diversity at neutrally-evolving sites in close proximity to the targets of selection (hitchhiking effect [21]). The extent to which linked selection affects neighboring sites depends on the

72 nucleotide diversity are expected to occur in genomic regions enriched for functional elements and with 73 lower recombination rates. A correlation between nucleotide diversity, gene density, and recombination 
74 rate is therefore indicative that linked selection is at play. Quantifying covariance between evolutionarily

75 independent species can help understand the interplay between these various conserved features of the

76 genome and their impact on patterns of diversity and differentiation along the genome.

We aim to address drift-selection dynamics during the Pleistocene climatic cycles by estimating the impact of demography and linked selection on the genome of Downy (Dryobates pubescens) and Hairy (D. villosus) Woodpeckers, two co-distributed species that share similar ecologies and evolutionary histories. Downy and Hairy Woodpecker are year-round residents of a variety of habitats in North America, occurring in sympatry across an exceptionally broad geographic area from Alaska to Florida, although the range of the Hairy Woodpecker extends further south, reaching portions of Central America and the Bahamas [45]. Despite looking very similar, the two species are not sisters and share a common ancestor more than eight million years ago, without any evidence of recent hybridization [46,47]. During the glacial cycles of the Pleistocene, especially when the polar ice sheets reached their maximum extent (Last Glacial Maximum; 21 kya), a large portion of the present-day distribution of Downy and Hairy Woodpeckers were covered in ice, and populations of both species were restricted to southern refugia $[12,48,49]$. After the retreat of Pleistocene glaciers, Downy and Hairy Woodpeckers extended their distributions north, recolonizing higher latitudes. Phylogeographical studies in Downy and Hairy Woodpecker revealed that populations currently inhabiting previously glaciated areas show strong signatures of population expansion and population structuring consistent with multiple glacial refugia [12,48-51]. This shared demographic history provides an opportunity to investigate multiple genomic factors that might have impacted the distribution of diversity across populations and within the genomes of these two natural evolutionary replicates.

In this study, we generated whole-genome resequencing data for Downy and Hairy Woodpeckers to test whether the heterogeneous genomic landscape of diversity and differentiation in both taxa is correlated with intrinsic features of the genome, such as recombination rate and gene density, and whether differences in demographic history had an impact on the efficacy of selection. We hypothesize that if linked selection reduced diversity at linked neutral sites along the genome, local levels of nucleotide diversity should be correlated with the rate of recombination and the density of targets of selection. In addition, we predict that if the efficiency of selection is a function of the demographic trajectory of populations during the Ice Age, large and more stable populations (i.e., larger long-term $\mathrm{N}_{\mathrm{e}}$ ) will exhibit lower genetic load and a stronger correlation between nucleotide diversity and intrinsic genomic properties, such as recombination rate. These results have implications for our understanding of the relative importance of

105 neutral and selective processes on the evolution of the genomic landscape of species heavily impacted by 106 glaciations. 


\section{Results}

108 Congruent population structure and genetic diversity

109 We characterized population genetic structure in Downy and Hairy Woodpeckers across an array of 110 ecological zones that would have been subject to varying effects of Pleistocene climatic cycles. We 111 collected whole genomes of 70 individuals each of Downy and Hairy Woodpecker (140 total samples; 112 Table S1), representing seven geographic locations in North America: Northeast (NE), Southeast (SE), 113 Midwest (MW), Southern Rockies (SR), Northern Rockies (NR), Pacific Northwest (NW), and Alaska 114 (AK; Figure 1a-b). Sequenced reads were mapped to a pseudo-reference genome of Downy Woodpecker 115 [52], yielding an average sequencing depth of 5.1x (1.4-12.5x) for Downy Woodpecker and 4.5x (1.1$11611.7 x)$ for Hairy Woodpecker. A total of 16,736,465 and 15,463,356 single nucleotide polymorphisms

117 (SNPs) were identified in the Downy and Hairy Woodpecker genomes, respectively, using the genotype 118 likelihood approach implemented in ANGSD [53]. 

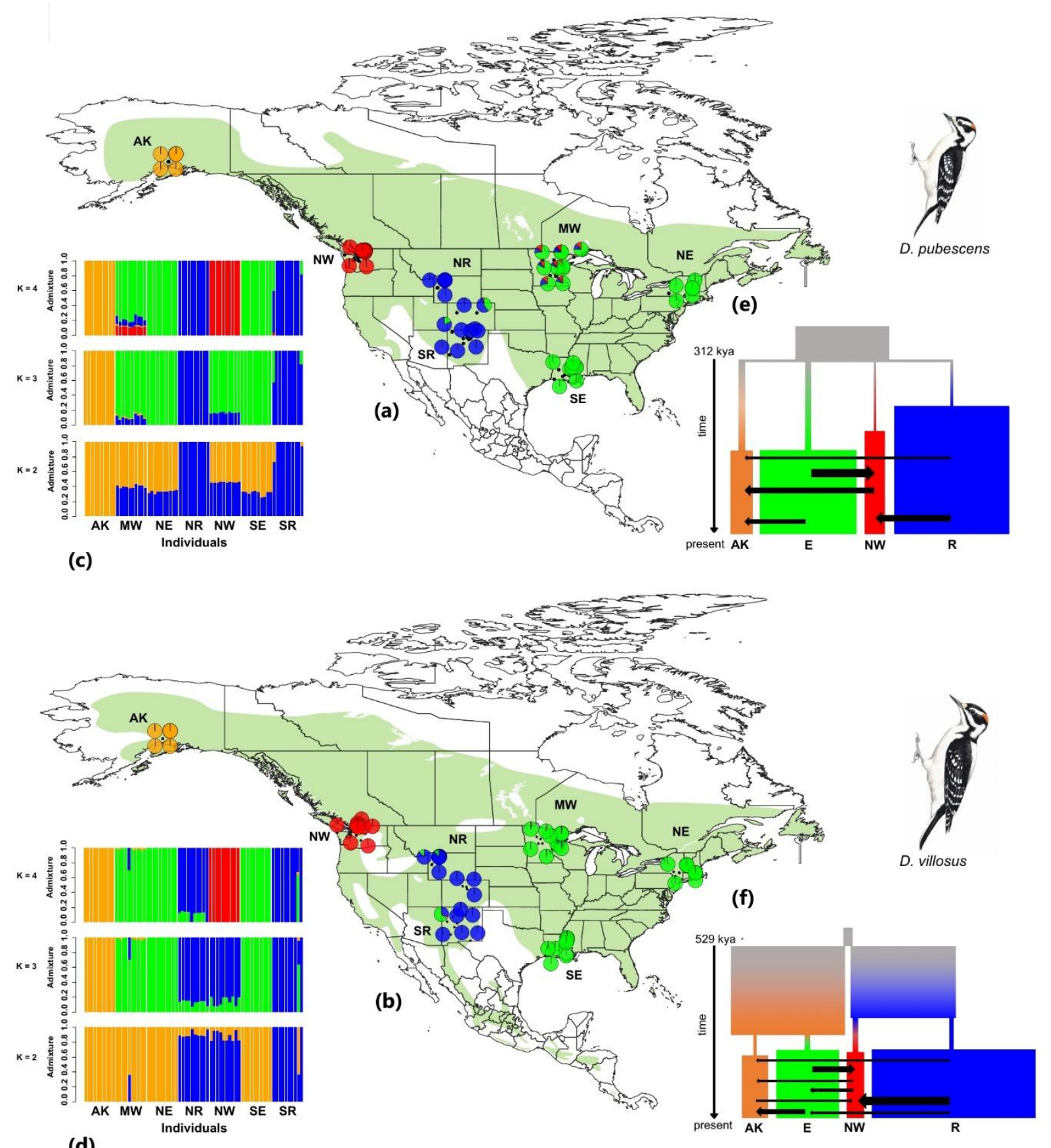

(d) for the $\mathrm{K}=2-4$. Each bar indicates an individual's estimated ancestry proportion for each genetic cluster, represented by different colors. (c-d) Map indicating the current range of Downy and Hairy Woodpecker (green shade), the locality of the samples, and their respective admixture proportions from NGSadmix (pie charts). (e-f) The best-fit demographic models from fastsimcoal2. The width of the rectangles and arrows are scaled relative to the estimated effective population sizes in haploid individuals $\left(\mathrm{N}_{\mathrm{e}}\right)$ and the migration rate $(m)$ in fraction of haploid individuals from donor population per generation, respectively. Only the values of migration rate $>10^{-7} \mathrm{x} \mathrm{N}_{\mathrm{e}}$ migrants per generation are shown. Illustrations reproduced with 
To assess patterns of genetic differentiation among these broadly distributed populations, we first performed a principal component analysis (PCA) on a subset of 71,229 and 71,816 independently-evolving

132 (linkage disequilibrium $r^{2}<0.2$ ) SNPs for Downy and Hairy Woodpecker, respectively. The three first

133 principal components (PCs) explained together 7.5\% (Downy) and 8.3\% (Hairy) of the total genetic

134 variance. We recovered congruent genetic structure across both species' ranges (Figure 2a,c). Geographic

135 structure was generally characterized by a genetic discontinuity between boreal-eastern and western

136 populations. In Downy Woodpecker, however, the Pacific Northwest population fell more closely related

137 to the Eastern group than the Western group (Figure 2a). Consistent with these findings, NGSadmix [54]

138 supported four geographically congruent genetic clusters $(\mathrm{K}=4)$ in the Downy and Hairy Woodpecker: East

139 (NE, SE, and MW), Pacific Northwest (NW), Rocky Mountains (SR and NR), and Alaska (AK; Figure 1c-

140 d). The average genome-wide estimate of $F_{\mathrm{ST}}$ was slightly larger in Hairy Woodpecker (average $\mathrm{F}_{\mathrm{ST}}=0.1$;

$1410.03-0.19$ ) than Downy Woodpecker (average $\mathrm{F}_{\mathrm{ST}}=0.08 ; 0.03-0.16$ ), indicating larger (but overlapping)

142 levels of population differentiation. In both species, the largest values of $\mathrm{F}_{\mathrm{ST}}$ involved comparisons between

143 Alaska and other populations (Downy: $F_{\text {ST }}\left[\right.$ AK vs NR] $=0.16$; Hairy: $F_{S T}[A K$ vs SR $]=0.19$ ), and the

144 lowest were within the East and the Rocky Mountains clusters (Downy: $F_{\mathrm{ST}}=0.03-0.06$; Hairy: $\mathrm{F}_{\mathrm{ST}}=0.03-$

145 0.04). 
(a)

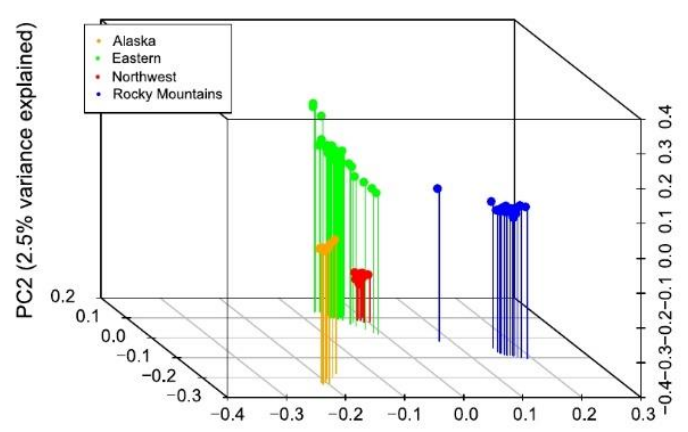

(c)

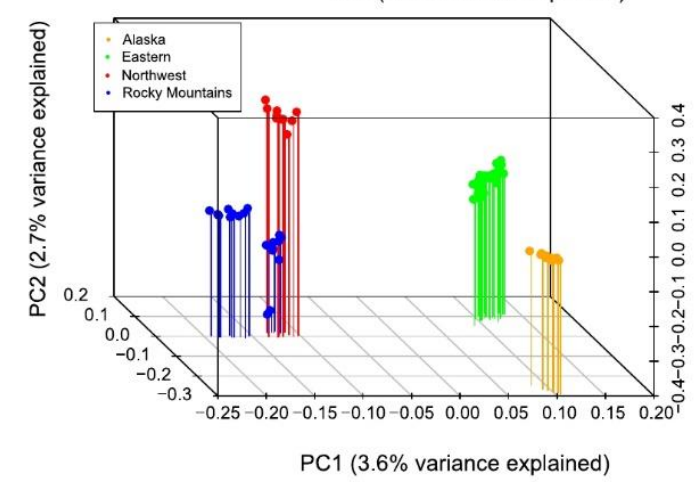

(b)

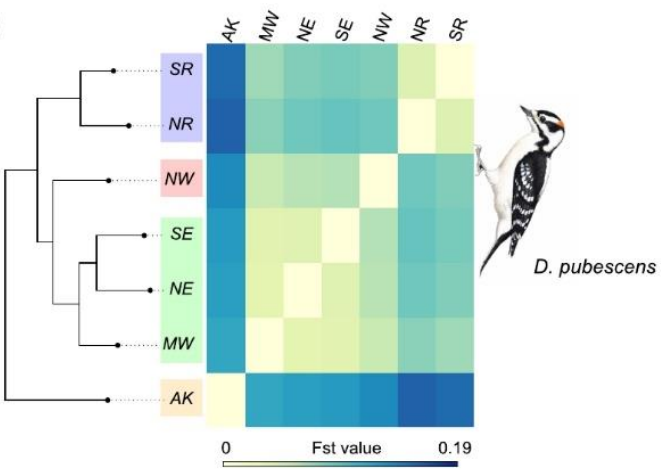

(d)

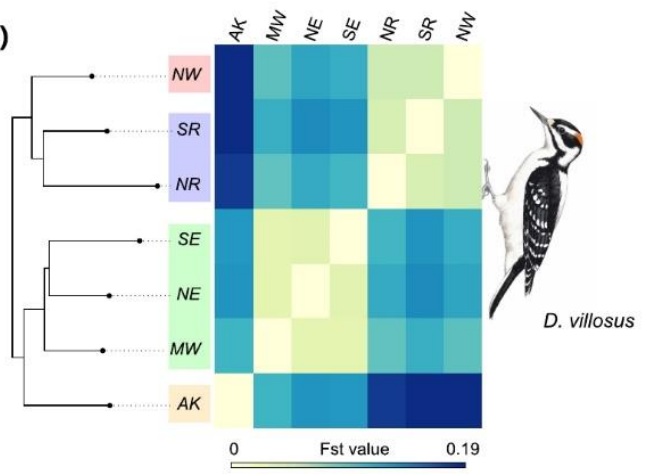

Figure 2. Population genetic structure in the Downy (top) and Hairy (bottom) Woodpecker. (a,c) Principal component analysis (PCA) of Downy and Hairy Woodpecker based on 71,228 and 71,763 unlinked genome-wide SNPs, respectively, with $<25 \%$ missing data and a minor allele frequency (maf) $>$ 0.05. (b,d) Heatmap showing genome-wide pairwise $\mathrm{F}_{\mathrm{ST}}$ values (left) and associated maximum likelihood tree based on the polymorphism-aware phylogenetic model (PoMo) in IQ-Tree 2. All nodes show 100\% bootstrap support. Darker colors on the heatmap correspond to larger values of $\mathrm{F}_{\mathrm{ST}}$. Illustrations reproduced with permission from Lynx Edicions.

Because the expansion and contraction of glaciers were expected to impact population structuring across the landscape, we explored spatial patterns of gene flow using the estimated effective migration surface (EEMS [55]). EEMS compares pairwise genetic dissimilarity among localities to identify geographic areas that deviate from the null expectation of isolation by distance (IBD). In both species, we detected a pronounced reduction in effective migration near the Great Plains and along the Rocky Mountains, especially in its Northern portion. In contrast, eastern North America showed a higher degree of connectivity when compared to the west (Figure 3). This finding indicates that major topographic features and variation in habitat availability contributed to the maintenance of population differentiation, despite the presence of gene flow. 

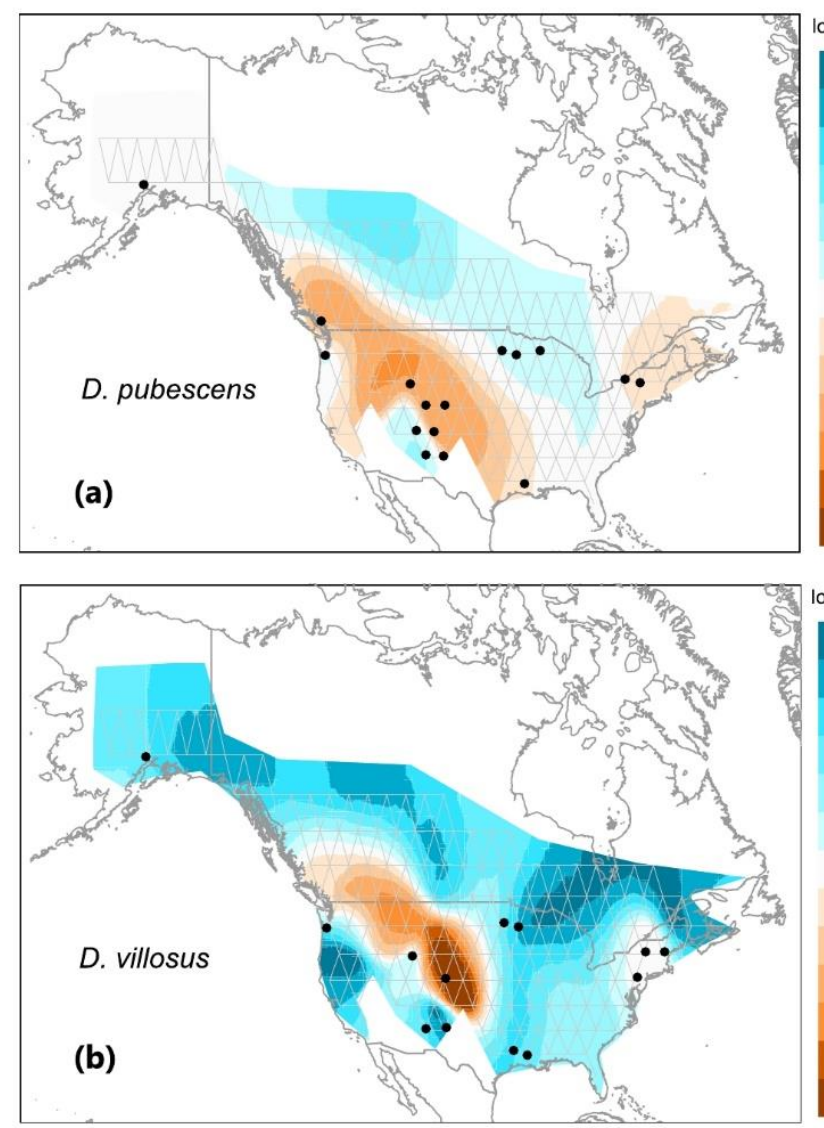
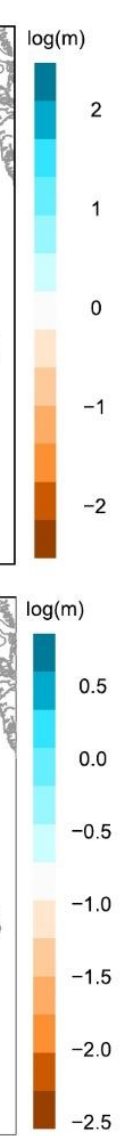

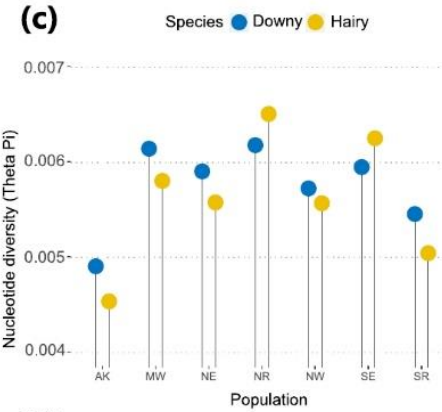

(d) Species पDowny Hairy

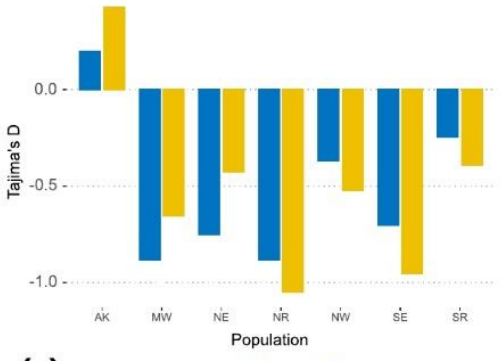

(e) Species Downy Hairy

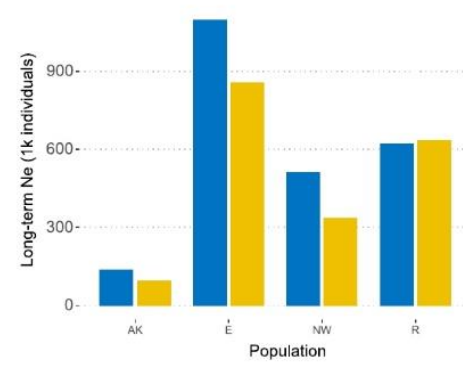

We tested for signatures of Quaternary climatic oscillations on population dynamics of Downy and Hairy

Figure 3. Spatial patterns of gene flow and genome-wide genetic variation in Downy and Hairy Woodpecker. (a) Effective migration surface inferred by EEMS in Downy Woodpecker and (b) Hairy Woodpecker. Warmer colors indicate lower and colder colors indicate higher effective migration (on a $\log$ scale) relative to the overall migration rate over the species range. Triangles represent the grid chosen to assign sampling locations to discrete demes. (c) Genome-wide pairwise nucleotide diversity $\left(\theta_{\pi}\right)$ per population. (d) Genome-wide Tajima's D per population. (e) The harmonic mean of effective population size $\left(\mathrm{N}_{\mathrm{e}}\right)$ estimated over the past one million years with Stairway Plot 2 for all four genetic clusters.

\section{Demographic history}

Woodpecker by assessing changes in $\mathrm{N}_{\mathrm{e}}$ over time and estimating demographic parameters. First, we employed Stairway Plot 2 [56] to infer fluctuations in $\mathrm{N}_{\mathrm{e}}$ over the past 500k years in each of the four detected genetic clusters assumed to represent panmictic populations. Stairway Plot 2 uses the site frequency spectrum (SFS) to fit a flexible multi-epoch model of changes in population size. For all 
demographic analyses, we used the folded SFS and specified a mutation rate of $4.007 \times 10^{-9}$ mutations per site per generation and a generation time of one year for both species [57]. Changes in effective population size over time were generally consistent between both species, being characterized by recurrent episodes of bottleneck followed by population expansion (Figure $4 \mathrm{a}-\mathrm{b}$ ). We found that within each genetic cluster, nucleotide diversity was highly correlated with the harmonic mean of the $\mathrm{N}_{\mathrm{e}}$ estimated from Stairway Plot 2 over the past 500 kya (long-term $\mathrm{N}_{\mathrm{e}}$; linear regression: $\mathrm{t}=4.876 ; \mathrm{R}^{2}=0.76 ; \mathrm{p}<0.002$; Figure $\mathrm{S} 1$ ), indicating these independent analyses were consistent.

(a)

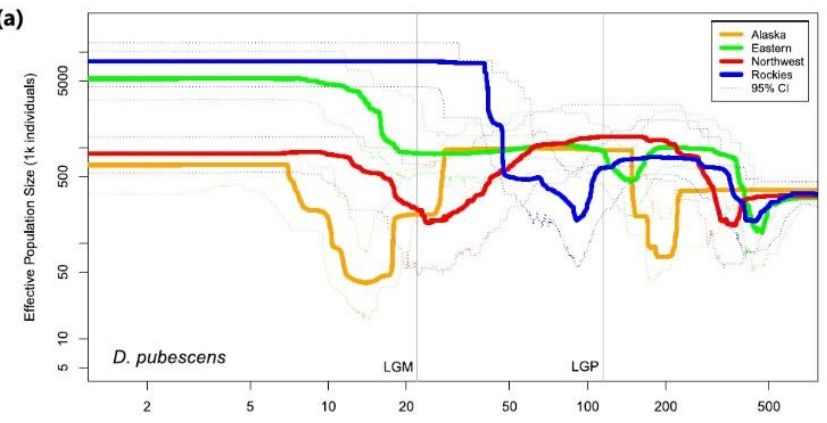

(b)

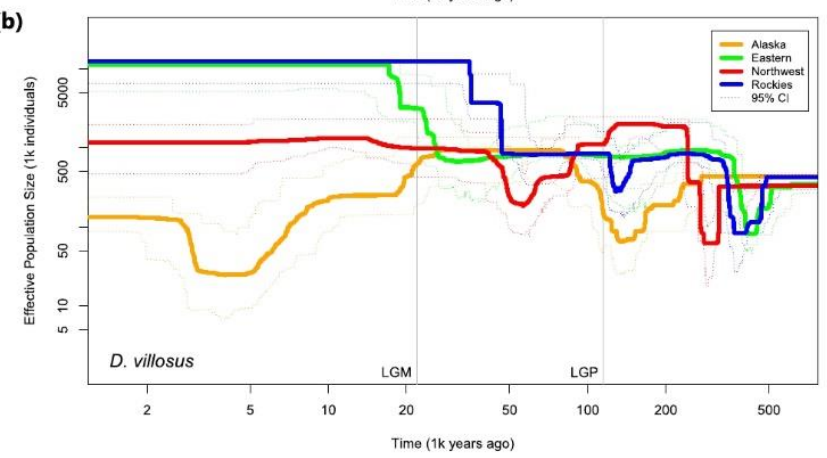

(c)

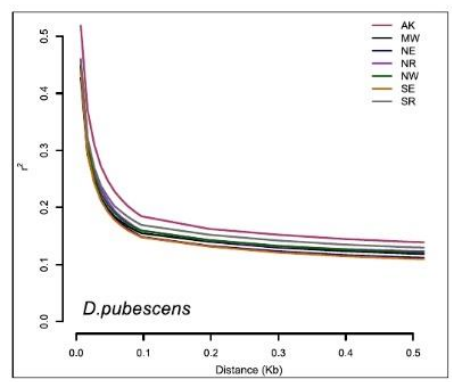

(d)

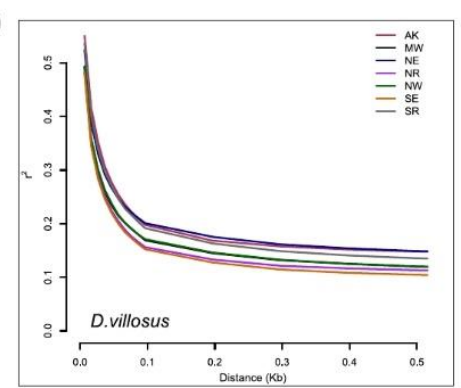

Figure 4. Changes in effective population size $\left(\mathrm{N}_{\mathrm{e}}\right)$ over time and linkage disequilibrium (LD) in Downy (top) and Hairy (bottom) Woodpecker. (a-b) Inferred history of effective population size of all four genetic clusters in Downy (a) and Hairy Woodpecker (b) obtained with Stairway Plot 2 using the folded SFS. For this analysis, we specified a mutation rate of $4.007 \times 10^{-9}$ mutations per site per generation. Both axes are represented in a log scale. Dotted lines represent $95 \%$ confidence intervals, and vertical lines represent the Last Glacial Period (LGP; 115 kya) and the Last Glacial Maximum (LGM; 21 kya). (c-d) Decay of linkage disequilibrium (LD) in all seven populations of Downy (c) and Hairy (d) Woodpecker. 
TREE polymorphism-aware phylogenetic model (PoMo [58,59]). The topology for Hairy Woodpecker showed two distinct clades - an East + Alaska and a West clade. The tree for Downy Woodpecker, however, revealed a different topology. First, the Pacific Northwest population (NW) was more closely related to the eastern clade than to the western clade, supporting our PCA analysis. In addition, the Alaska (AK) population was sister to all other populations. Two hypotheses could explain this pattern: either (i) Alaska was a distinctive clade that differentiated from the other Downy Woodpecker populations as a consequence of persistence in a separate glacial refugium near Beringia, as has been suggested for other North American taxa $[2,60,61]$, or (ii) the topology of the Downy Woodpecker population tree was more reflective of other factors, such as patterns of gene flow and geographic distance among localities, as opposed to the actual order of population splits. If this was the case, then we expect the relationships among populations to better fit a polytomous tree rather than a bifurcating tree.

To test these alternative population histories, we used the SFS-based method fastsimcoal2 v2.6.0.3 [62] to estimate demographic parameters and evaluate the support for two alternative models - (i) a model where all populations diverge synchronously from a single ancestral refugium and expand independently with asymmetric gene flow, and (ii) a bifurcating model where populations diverge at different times from multiple refugia (e.g., Beringia and East or East and West) and expand independently with asymmetric gene flow, following the IQ-TREE topology. S2; Figure 1f), in which two ancestral populations (putatively located in East and West) diverged from each other around $529 \mathrm{kya}(95 \% \mathrm{CI}=513-561 \mathrm{kya}$; Table S3) and gave rise to the four genetic clusters, which then underwent strong bottlenecks. A final explosive expansion then occurred between 193-212 kya when populations grew up to 12-fold. In contrast, Downy Woodpecker showed support for model i, in which all populations diverge from a single major refugium (Table S2; Figure 1e). This divergence occurred around less than $10 \%$ of its original size in most populations. A final population expansion then occurred at the end of the Mid-Pleistocene (152-232 kya). Overall, estimates of $\mathrm{N}_{\mathrm{e}}$ from fastsimcoal2 confirmed the trends observed in Stairway Plot 2, albeit with less resolution. We found large and variable levels of postexpansion gene flow across populations in both species (Downy: 0-4.8 migrants per generation; Hairy: 06.66 migrants per generation) that confirmed our EEMS migration surfaces.

\section{Genomic correlates of nucleotide diversity and differentiation}

229 To elucidate the evolutionary processes shaping levels of genetic variation along the genome of Downy and 230 Hairy Woodpecker, we investigated the correlation between regional levels of nucleotide diversity, 
231 measured across non-overlapping $100 \mathrm{~kb}$ windows, and three genomic features: recombination rate, gene

232 density, and base composition. We found that nucleotide diversity varied widely along the genome $\left(\theta_{\pi \text { Downy }}\right.$

$233=7.5 \times 10^{-4}-1.9 \times 10^{-2} ; \theta_{\pi \text { Hairy }}=1.1 \times 10^{-3}-2.2 \times 10^{-2}$ ), but this variation was highly correlated between

234 Downy and Hairy Woodpecker (Pearson's $r=0.9 ; \mathrm{p}<0.001$; Figure S2). To estimate recombination rates,

235 we used ReLERNN [63], a method that uses a machine-learning approach to infer per-base recombination

236 rates. We found recombination rates to be highly correlated between the two species (Pearson's $r=0.66 ; \mathrm{p}$

$237<0.001)$. Across the genome, we estimated a mean per-base recombination rate $(r)=2.42 \times 10^{-9} \mathrm{c} / \mathrm{bp}(0$

$\left.2383.87 \times 10^{-9}\right)$ in Downy Woodpecker and $r=3.69 \times 10^{-9} \mathrm{c} / \mathrm{bp}\left(4.85 \times 10^{-10}-3.87 \times 10^{-9}\right)$ in Hairy Woodpecker.

239 Considering the average long-term $\mathrm{N}_{\mathrm{e}}$ of Downy and Hairy Woodpecker as approximately $1 \times 10^{6}$ in the

240 East population, these recombination rates correspond to a population-scaled rate $\rho=4 \mathrm{~N}_{\mathrm{e}} \mathrm{r}=0.008$ and

2410.012 , respectively. Mean recombination rates were 2-3-fold higher in autosomal chromosomes compared

242 to the sex-linked Z chromosome (Figure S3-4), consistent with differences in $\mathrm{N}_{\mathrm{e}}$ between sex chromosomes

243 [64-66]. As a result of both high recombination rates and large $\mathrm{N}_{\mathrm{e}}$, we also observed that linkage

244 disequilibrium (LD) in Downy and Hairy Woodpecker decays very rapidly. LD drops to half of its initial

245 levels in less than $100 \mathrm{bp}$ (Figure 5c-d). Consistently, the average LD was greater for populations with

246 smaller $\mathrm{N}_{\mathrm{e}}$ or populations that have likely experienced a more recent founder event, such as Alaska and the

247 Southern Rockies. We found a significant positive association between nucleotide diversity $\left(\theta_{\pi}\right)$ and

248 recombination rates in both species (linear regression - Downy: $\mathrm{t}=47.67, \mathrm{R}^{2}=0.165, \mathrm{p}<0.001$; Hairy: $\mathrm{t}$

$249=54.17, \mathrm{R}^{2}=0.204, \mathrm{p}<0.001 ;$ LOESS regression - Downy: $\operatorname{span}=0.5, \mathrm{R}^{2}=0.207$; Hairy: $\operatorname{span}=0.5, \mathrm{R}^{2}$

$250=0.207$; Figure 5a-b). This association, however, is expected (to a certain extent) even if diversity is not

251 correlated with recombination rates because recombination rates are estimated directly from $\theta_{\mathrm{w}}$. 

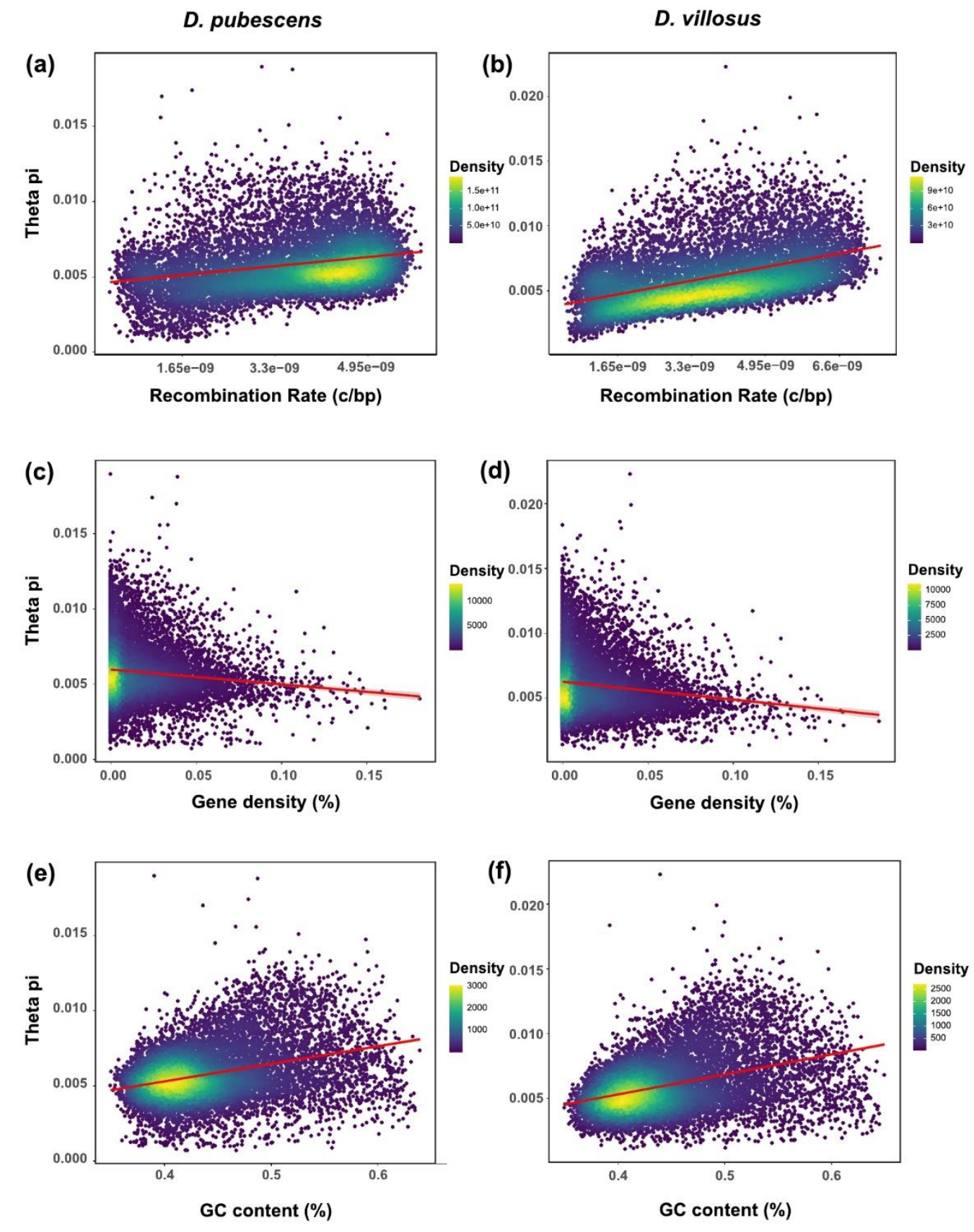

Figure 5. Genomic predictors of nucleotide diversity in Downy (left) and Hairy (right) Woodpecker. Association between nucleotide diversity $\left(\theta_{\pi}\right)$ and three features of the genome: (a-b) recombination rates (Downy: $\mathrm{t}=47.67, \mathrm{p}<0.001$; Hairy: $\mathrm{t}=54.17, \mathrm{p}<0.001$ ), $(\mathbf{c}-\mathbf{d})$ gene density (Downy: $\mathrm{t}=-12.03, \mathrm{p}$ $<0.001$; Hairy: $\mathrm{t}=-14.89, \mathrm{p}<0.001$ ), and (e-f) GC content (Downy: $\mathrm{t}=36.37, \mathrm{p}<0.001$; Hairy: $\mathrm{t}=44.16$, $\mathrm{p}<0.001$ ). Each point in the scatter plot represents a $100 \mathrm{~kb}$ window of the genome. Colors indicate the density of points. 
To further investigate the impact of linked selection on the genomic landscape of diversity, we also tested the prediction that regions of the genome with a higher density of targets of selection (i.e., genes) exhibit lower nucleotide diversity. Gene density was measured as the percentage of coding sequence in each of the $100 \mathrm{~kb}$ windows. Our results revealed a weak but significant negative association between nucleotide diversity $\left(\theta_{\pi}\right)$ and gene density (linear regression - Downy: $t=-12.03, R^{2}=0.0123, p<0.001$; Hairy: $\mathrm{t}=-14.89, \mathrm{R}^{2}=0.0189, \mathrm{p}<0.001$; LOESS regression - Downy: span $=0.5, \mathrm{R}^{2}=0.0139$; Hairy: span $=0.5, \mathrm{R}^{2}=0.021 ;$ Figure $\left.5 \mathrm{c}-\mathrm{d}\right)$. This association was not driven by the collinearity between gene density and recombination because this correlation was positive and negligible (Downy: Pearson's $r=$ $0.045, \mathrm{p}<0.001$; Hairy: Pearson's $r=0.032, \mathrm{p}<0.001)$. We also found that regions with high GC content tended to show higher nucleotide diversity (linear regression - Downy: $t=36.37, \mathrm{R}^{2}=0.0123, \mathrm{p}<0.001$; Hairy: $\mathrm{t}=44.16, \mathrm{R}^{2}=0.145, \mathrm{p}<0.001$; Figure $\left.5 \mathrm{e}-\mathrm{f}\right)$. GC content, however, was positively correlated with gene density in both species (Downy: Pearson's $r=0.25 ; \mathrm{p}<0.001$; Hairy: Pearson's $r=0.25 ; \mathrm{p}<0.001$; Figure S5-6) and weakly correlated with recombination rates in Hairy Woodpecker (Pearson's $r=0.064$; $\mathrm{p}<0.001$; Figure S5-6). We then performed a principal component regression (PCR) to separate the effect of individual explanatory variables and control for the multicollinearity among predictor variables. Principal component regression summarizes variables into orthogonal components (PCs) and uses these components as predictors in a linear regression. PC2, which represented almost exclusively recombination rates (Table 1), uniquely explained $12.3 \%$ and $18.6 \%$ of variation in nucleotide diversity in Downy and Hairy Woodpecker, respectively (PC2 linear regression - Hairy: $t=51.1, R^{2}=0.186, p<0.001$; Downy: $t$ $\left.=40.14, \mathrm{R}^{2}=0.123, \mathrm{p}<0.001\right)$. Both PC1 and PC3 represented the correlation between gene density and GC content, but PC3 had a much stronger effect (Table 1), accounting for $14.4 \%$ and $15.5 \%$ of the variation in nucleotide diversity in Downy and Hairy Woodpecker, respectively (PC3 linear regression - Downy: $t$ $=45.92, \mathrm{R}^{2}=0.155, \mathrm{p}<0.001$; Hairy: $\left.\mathrm{t}=43.97, \mathrm{R}^{2}=0.144, \mathrm{p}<0.001\right)$. Considering that gene density and GC content had an equal contribution to PC3 (Table 1), we were unable to differentiate their relative contributions to the relationship. Regardless, our analyses confirm the central role that these genomic properties played in shaping patterns of nucleotide diversity along the genome. 
Table 1. Principal component regression.

\begin{tabular}{c|c|c|c|c}
\hline \multirow{2}{*}{ Species } & Explanatory variables & \multicolumn{3}{|c}{$\%$ of variance explained $\left(\mathbf{R}^{\mathbf{2}}\right)$} \\
\cline { 3 - 5 } & & PC1 & PC2 & PC3 \\
\hline $\begin{array}{c}\text { Downy } \\
\text { Woodpecker }\end{array}$ & Recombination rate & 0.08 & 11.89 & 0.11 \\
& Gene density & 1.7 & 0.03 & 7.8 \\
& GC content & 1.65 & 0.36 & 7.58 \\
& Total & 3.45 & 12.3 & 15.51 \\
\hline Hairy Woodpecker & Recombination rate & 0.35 & 17.35 & 0.01 \\
& Gene density & 2.84 & 1.02 & 7 \\
& GC content & 2.96 & 0.06 & 7.34 \\
& Total & 6.18 & 18.6 & 14.47 \\
\hline
\end{tabular}

The effect of linked selection is expected to be weaker in populations that underwent more severe bottlenecks due to their smaller long-term $\mathrm{N}_{\mathrm{e}}$ when compared to stable populations that maintained large $\mathrm{N}_{\mathrm{e}}[67,68]$. We tested this prediction by quantifying the strength of correlation between nucleotide diversity $\left(\theta_{\pi}\right)$ and gene density in all four genetic clusters of Downy and Hairy Woodpecker which showed varied demographic responses to the Pleistocene glaciations. We found that long-term $\mathrm{N}_{\mathrm{e}}$ predicted the coefficient of correlation between genetic diversity and the density of targets of selection (Table 2). Alaska, for example, showed the weakest correlation (Downy: Pearson's $r=-0.1008, \mathrm{t}=-10.8, \mathrm{p}<0.001$; Hairy: Pearson's $r=-0.1083, \mathrm{t}=-11.6, \mathrm{p}<0.001$ ), whereas Rocky Mountains showed the strongest (Downy: Pearson's $r=-0.1106, \mathrm{t}=-11.9, \mathrm{p}<0.001$; Hairy: Pearson's $r=-0.1351, \mathrm{t}=-14.5, \mathrm{p}<0.001)$. Although the differences in coefficients were small, these results support the expectation that different demographic trajectories affect the efficacy of natural selection owing to differences in levels of genetic drift. 
Table 2. Strength of correlation between nucleotide diversity $\left(\theta_{\pi}\right)$ and gene density across the four genetic clusters of Downy and Hairy Woodpecker.

\begin{tabular}{|c|c|c|c|c|}
\hline \multirow[t]{2}{*}{ Populations } & \multicolumn{2}{|c|}{ Downy Woodpecker } & \multicolumn{2}{|c|}{ Hairy Woodpecker } \\
\hline & Pearson's r & t-value & Pearson's r & t-value \\
\hline $\mathrm{AK}$ & $-0.1008^{*}$ & -10.867 & $-0.1083^{*}$ & -11.643 \\
\hline NW & $-0.1007^{*}$ & -10.847 & $-0.1384^{*}$ & -12.966 \\
\hline $\mathrm{E}$ & $-0.1077^{*}$ & -11.618 & $-0.1215^{*}$ & -13.084 \\
\hline $\mathrm{R}$ & $-0.1106^{*}$ & -11.927 & $-0.1351^{*}$ & -14.571 \\
\hline
\end{tabular}

Because genomic properties are also expected to impact levels of population differentiation across the genome, we also tested the association between nucleotide diversity, recombination rate, and the average intraspecific population differentiation $\left(\mathrm{F}_{\mathrm{ST}}\right)$ across non-overlapping $100 \mathrm{~kb}$ windows. For each window, we calculated the $F_{S T}$ between each pair of populations and summarized the global $F_{S T}$ landscape using two approaches: (i) the average $\mathrm{F}_{\mathrm{ST}}$ across all population pairs; and (ii) the first principal component (PC1) explaining most of the variation in pairwise $\mathrm{F}_{\mathrm{ST}}$ (Downy: variance explained $=37.51 \%$; Hairy: variance explained $=47.5 \%$ ). Summaries of $\mathrm{F}_{\mathrm{ST}}$ produced by these two approaches were highly correlated (Downy: Pearson's $r=0.97 ; \mathrm{p}<0.001$; Hairy: Pearson's $r=0.98 ; \mathrm{p}<0.001$ ), so we only considered the average $\mathrm{F}_{\mathrm{ST}}$ for simplicity. There was considerable variation in $\mathrm{F}_{\mathrm{ST}}$ along the genome (Downy: $\mathrm{F}_{\mathrm{ST}}=0.01-$ 0.25; Hairy: $\mathrm{F}_{\mathrm{ST}}=0.01-0.32$ ), indicating high variability in patterns of population differentiation. We recovered a significant negative association between average $\mathrm{F}_{\mathrm{ST}}$ and nucleotide diversity, suggesting that areas of genome that show elevated differentiation tend to be characterized by reduced diversity (linear regression - Downy: $\mathrm{t}=-19.12, \mathrm{R}^{2}=0.03 ; \mathrm{p}<0.001$; Hairy: $\mathrm{t}=-53.49, \mathrm{R}^{2}=0.2 ; \mathrm{p}<0.001$; Figure 6). Finally, we found a negative association between average $\mathrm{F}_{\mathrm{ST}}$ and recombination rates, indicating higher differentiation in regions of low recombination (linear regression - Downy: $t=-32.18, \mathrm{R}^{2}=0.08 ; \mathrm{p}<0.001$; Hairy: $\left.\mathrm{t}=-41.55, \mathrm{R}^{2}=0.13 ; \mathrm{p}<0.001\right)$. 

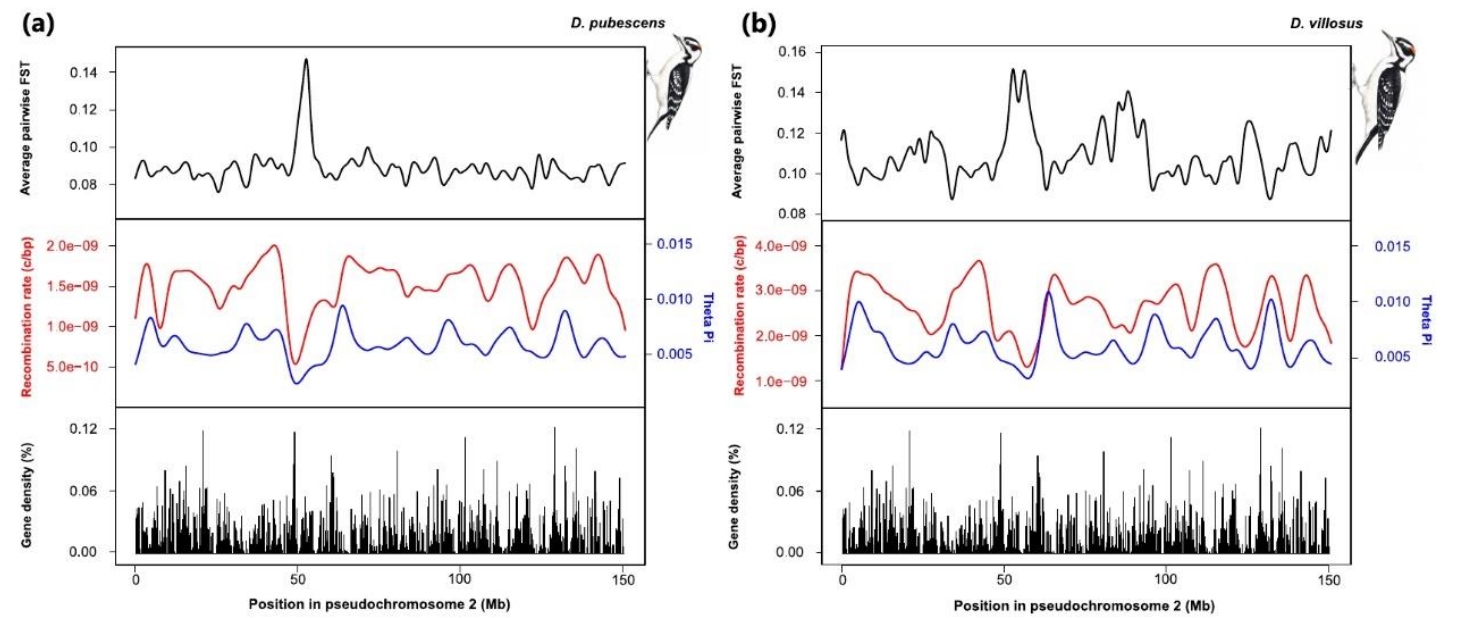

Figure 6. Landscape of diversity and differentiation of chromosome 2 of Downy (a) and Hairy (b) Woodpecker for illustration. Top plot shows the average pairwise $\mathrm{F}_{\text {ST }}$ calculated across non-overlapping $100 \mathrm{~kb}$ windows. Middle plot indicates the recombination rate in $\mathrm{c} / \mathrm{bp}$ (red) and the nucleotide diversity $\left(\theta_{\pi}\right.$; blue) for each non-overlapping $100 \mathrm{~kb}$ window. Bottom plot represents the percentage of coding sequence in each non-overlapping $100 \mathrm{~kb}$ window. Illustrations reproduced with permission from Lynx Edicions.

Genetic load and the efficacy of selection

To further explore the magnitude of linked selection in the genome of Downy and Hairy Woodpecker, we classified each variant according to their functional impact as predicted by the gene annotation. We found that the majority of identified SNPs in Downy and Hairy Woodpecker were classified as modifiers (Downy: 99.35\%; Hairy: 99.13\%), which are variants in intergenic or intronic regions whose impacts are hard to determine but tend to be neutral to nearly neutral. Low impact variants (i.e., synonymous mutations) characterized $0.46 \%$ and $0.64 \%$ of SNPs in Downy and Hairy Woodpecker, respectively. Moderate impact variants, mutations that cause a change in amino acid sequence (i.e., nonsynonymous mutations) represented $0.17 \%$ and $0.22 \%$ of the SNPs in Downy and Hairy Woodpecker, respectively. Finally, only $0.006 \%$ and $0.007 \%$ of the SNPs were classified as high impact in Downy and Hairy Woodpecker, respectively. These variants correspond to mutations that cause loss of function, such as loss or gain of a start or stop codon and are therefore expected to occur at very low frequencies.

We investigated differences in the burden of deleterious alleles carried by populations of Downy and Hairy Woodpecker that could reflect differences in the efficacy of purifying selection. For this analysis, 
could be determined unambiguously. Our results revealed that the frequency distribution of mutations with moderate and high impact shifted downwards compared to the mutations with low impact (Figure 7a-b). This indicates that purifying selection was successful in purging mutations that were highly deleterious.

362 Hairy Woodpecker, however, showed a larger excess of low frequency mutations of high impact when

363 compared to Downy Woodpecker (Figure 7a-b), suggesting that purifying selection might have been more

364 efficient in Hairy Woodpecker. To further evaluate whether the efficacy of purifying selection varied across

365 populations with different demographic trajectories, we estimated for each individual the genetic load as

366 the ratio of the count of homozygous derived alleles of high impact (i.e., highly deleterious) over the count

367 of homozygous derived alleles of low impact (i.e., synonymous). This metric is a proxy for the genetic load

368 under a recessive model while controlling for the underlying population differences in the neutral SFS

$369[69,70]$. We also computed the same metric considering an additive model, in which the presence of a single

370 copy of the derived allele has fitness consequences. Our results reveal that the recessive deleterious load

371 was overall larger in Downy than Hairy Woodpecker, but this difference was not statistically significant

372 (Kruskal-Wallis $\chi^{2}=1.33, \mathrm{df}=1, \mathrm{p}=0.24$; Figure $7 \mathrm{c}-\mathrm{d}$ ). The recessive deleterious load was much larger in

373 the Rocky Mountains when compared to other populations. Alaska also showed elevated recessive

374 deleterious load in both species, generally larger than the East and Pacific Northwest (Figure 7c-d). Overall,

375 these findings do not support the prediction that populations with stronger bottlenecks exhibit high

376 deleterious load. 
(a)

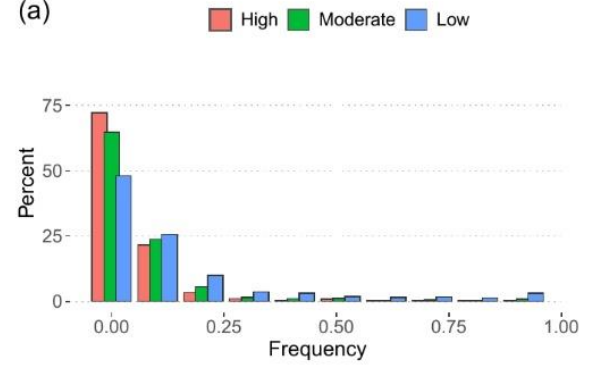

(c)

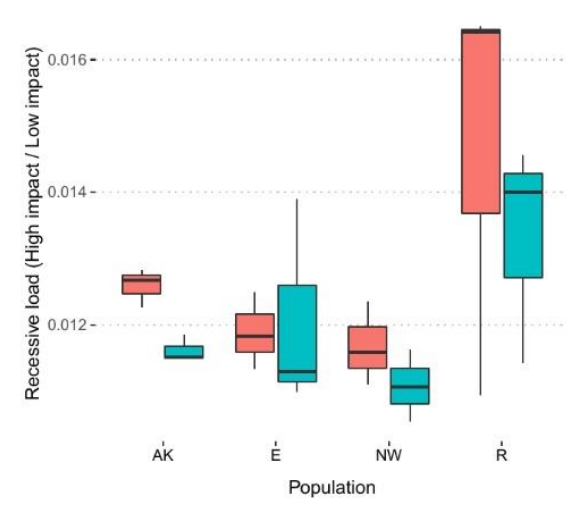

(b)

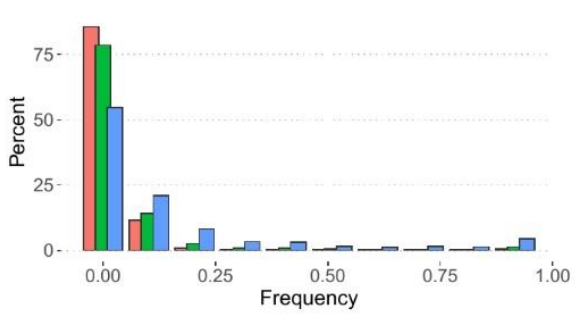

(d) 追 Downy 追 Hairy

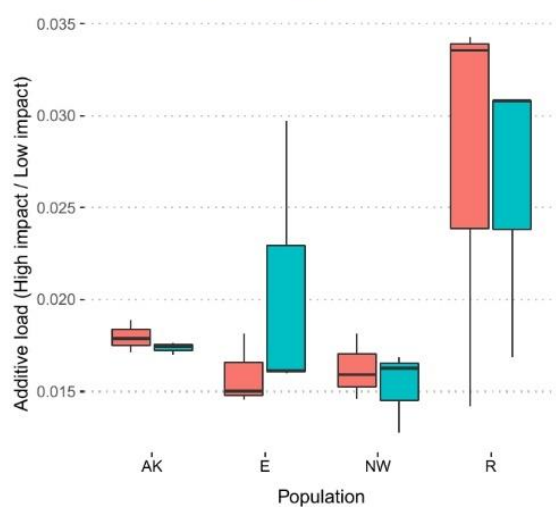

Figure 7. Deleterious load in Downy and Hairy Woodpecker. (a) Site frequency spectrum (SFS) for variants with low (neutral), moderate (mild), and high (deleterious) impact in Downy Woodpecker and (b) Hairy Woodpecker. (c) Ratio of homozygous derived variants of high impact (deleterious) over homozygous derived variants of low impact (neutral) in each genetic cluster and species (recessive model). (d) Ratio of the total number of derived variants of high impact (deleterious) over total number of derived variants of low impact (neutral) in each genetic cluster and species (additive model). Horizontal bars denote population medians.

Lastly, we investigated the overall impact of natural selection on protein-coding sequences of Downy and Hairy Woodpecker. We calculated the ratio of synonymous over nonsynonymous substitutions (dN/dS) along the branches leading to Downy and Hairy Woodpecker using a set of 397 high-quality orthologous genes distributed throughout the genome. $\mathrm{dN} / \mathrm{dS}$ ratio was higher in Downy Woodpecker $(\mathrm{dN} / \mathrm{dS}=0.065)$ than in Hairy Woodpecker $(\mathrm{dN} / \mathrm{dS}=0.053)$, suggesting that purifying selection might have been weaker in the Downy Woodpecker lineage over deeper evolutionary times (i.e. $>4 \mathrm{~N}_{\mathrm{e}}$ generations ago [71-73]). 


\section{Discussion}

397 Our genomic analyses reveal that both Ice Age demographic fluctuations and linked selection played a 398 significant role shaping patterns of diversity and differentiation across populations and along the genomes of Downy and Hairy Woodpecker. We found that genome-wide nucleotide diversity, as well as the landscape of recombination, are highly correlated between these two species, which diverged more than 8 mya. Despite shared environmental pressures, this coupling suggests that intrinsic properties of the genome, such as recombination rate, might be conserved across deep evolutionary time. We posit that linked selection might underlie the genomic heterogeneity observed, as demonstrated by a significant association

404 between nucleotide diversity, recombination rate, and gene density. Despite strong fluctuations in $\mathrm{N}_{\mathrm{e}}$ over 405 the Pleistocene, Downy and Hairy Woodpecker maintained large population sizes, which might have 406 facilitated the action of natural selection. Nevertheless, given the large differences in long-term $\mathrm{N}_{\mathrm{e}}$ observed 407 among populations, our results indicate variation in the efficacy of selection.

408 Conserved properties of the genome underlie the correlated genomic landscape of Hairy and Downy 409 Woodpecker

410 We recovered large heterogeneity in patterns of nucleotide diversity $\left(\theta_{\pi}\right)$ and $\mathrm{F}_{\mathrm{ST}}$ along the genomes of 411 Downy and Hairy Woodpecker. Despite this variation, our results revealed a highly correlated genomic 412 landscape between the two species. Such covariation in levels of genome-wide measures of diversity and 413 differentiation across distantly related species is common [34,35,74-77] and suggests that properties of the 414 genome, such as mutation rate, recombination rate, and density of targets of selection are conserved across 415 deep evolutionary time [78]. For example, bird genomes are known to show large karyotypic stability, with 416 very few chromosomal rearrangements and high synteny across highly divergent species [79-82]. Features 417 of the genome, such as recombination rates and GC content, might also be conserved across species. We 418 found that estimates of recombination rate are highly correlated between Downy and Hairy Woodpecker, 419 although higher in the latter. Linkage disequilibrium (LD), which is a function of both recombination rate 420 and $\mathrm{N}_{\mathrm{e}}$, was extremely short in Downy and Hairy Woodpecker. Whereas linkage disequilibrium extends 421 for over thousands of base pairs in humans [83,84], for instance, it breaks after only 100 bp in Downy and 422 Hairy Woodpecker. Such properties have been observed in other bird species with very large $\mathrm{N}_{\mathrm{e}}[85,86]$. 423 We also found large variation in recombination rates both within and among chromosomes, with the $\mathrm{Z}$ 424 chromosome showing the lowest rates. Considering the lack of recombination across much of the $\mathrm{Z}$ 425 chromosome in female birds (heterogametic sex; $\mathrm{ZW}$ ), at the population level, crossing-over occurs at a 426 much lower rate in sex chromosomes than in their autosome counterparts $[64,87,88]$. Similar to Downy and 427 Hairy Woodpecker, recombination in the chicken (Gallus gallus) was approximately 2.5 times lower in the 
$\mathrm{Z}$ chromosome than in the autosomes [89,90]. As a consequence, many bird species show reduced diversity and faster divergence in the $\mathrm{Z}$ chromosome $[64,85,91,92]$.

One of the main mechanisms proposed to explain the substantial heterogeneity in levels of polymorphism along the genome is the effect of linked selection [21,23,24]. Both positive selection (i.e., in favor of a beneficial allele) and negative selection (i.e., against a deleterious allele) are expected to reduce diversity around functional elements $[21,23]$. Such a reduction is extended to all neighboring sites that happen to be linked to the target of selection (hitchhiking effect [21]). The extent to which adjacent sites are affected by linked selection is dependent on the recombination landscape, such that regions where recombination rate is lower tend to show lower genetic diversity and vice versa $[32,93,94]$. Similarly, the higher the density of functional elements (i.e., targets of selection), the more severe is the reduction in genetic diversity due to the effect of recurrent selection [33,38-40]. Although a correlation between nucleotide diversity and recombination may arise in the absence of linked selection, we do not expect that to be true for gene density, since directional selection unavoidably affects levels of polymorphism in regions presumed to be functional. Such correlations are therefore interpreted as evidence of the effect of selection on linked neutral sites and can be used to assess the magnitude of linked selection [24,95]. In light of these results, we identified strong evidence that linked selection has contributed to patterns of genetic diversity along the genomes of Downy and Hairy Woodpecker. First, nucleotide diversity $\left(\theta_{\pi}\right)$ was positively associated with recombination rates in both species. Second, there was a weak but highly significant association between nucleotide diversity $\left(\theta_{\pi}\right)$ and gene density. Third, as predicted by theory, the strength of association between nucleotide diversity $\left(\theta_{\pi}\right)$ and gene density varied according to the long-term $\mathrm{N}_{\mathrm{e}}$, such that larger populations showed more pronounced signatures of linked selection.

Natural selection is also expected to impact levels of genetic differentiation along the genome $[35,96,97]$. We estimated a weak but significant negative association between nucleotide diversity $\left(\theta_{\pi}\right)$ and the average pairwise $\mathrm{F}_{\mathrm{ST}}$, indicating that regions of the genome that are highly differentiated between populations tend to show reduced diversity. These correlations are consistent with the effect of linked selection continuously eroding diversity near targets of selection (especially in regions of low recombination), which leads to the inflation of local levels of population differentiation [96]. Because beneficial alleles are not expected to appear frequently, background selection against deleterious alleles is the most likely selective mechanism underlying the correlation between $\mathrm{F}_{\mathrm{ST}}$, nucleotide diversity, and recombination rate [97,98]. These findings suggest that population-specific selection associated with local adaptation (i.e., divergent selection) is not necessary to produce a correlated genomic landscape. Comparative analyses across both distantly and closely related bird species demonstrate that linked 
selection can reduce genetic diversity prior to population splits and consequently produce parallel patterns of genetic differentiation in regions of low recombination [75,77,98,99].

We found that population structure was spatially congruent between Downy and Hairy Woodpecker, but that their demographic histories and extent of genetic structuring varied. Both species were characterized by phylogeographic clusters that are consistent with previous studies $[12,48,49]$ and were concordant with structuring in glacial refugia, albeit forming during different time scales. The observation of common geographic patterns formed across different time periods highlights the predictability of the interaction of the physical landscape, drift, and gene flow on genetic diversity. Further evidence of the dramatic effects of Pleistocene climatic fluctuations on genetic diversity were the repeated cycles of population contraction and expansion. Yet, despite strong variation in $\mathrm{N}_{\mathrm{e}}$ over the past $500 \mathrm{ky}$, our data indicates that Downy and Hairy Woodpecker have been resilient enough to maintain relatively large populations, which favored the maintenance of very high genetic diversity, even in the face of repeated bottlenecks. While non-equilibrium population dynamics are a hallmark of species that occur in previously glaciated areas [2], the relationship

477 between the magnitude of Plesitocene population size reductions and the efficacy of selection under these conditions remain poorly explored in empirical systems.

Consistent with theoretical predictions, nucleotide diversity within populations was strongly correlated with the long-term $\mathrm{N}_{\mathrm{e}}$. Alaska showed the lowest genome-wide genetic diversity, likely as a consequence of being one of the latest areas to be deglaciated and most recently founded. On the other hand, the Northern Rockies exhibited the largest nucleotide diversity and long-term $\mathrm{N}_{\mathrm{e}}$, in both focal species. Data from multiple sources support the existence of a temporally fluctuating ice-free corridor along the Canadian Rocky Mountains that might have functioned as a glacial refugium [10,100-102]. Thus, it is possible that suitable habitat might have allowed rapid growth and persistence of large populations in the North Rockies during the glacial periods of the Pleistocene.

489 We investigated whether differences in the demographic trajectories of populations of Downy and Hairy 490 Woodpecker in response to the Pleistocene glaciation had an impact on the efficacy of natural selection 491 across the genome. Given that purifying selection is more efficient in larger populations [103], we 
more likely to have accumulated highly deleterious mutations (i.e., genetic load [26-30]). We failed to find support for this prediction. In contrast to our expectations, we found that the Rocky Mountains, the genetic cluster with the largest long-term $\mathrm{N}_{\mathrm{e}}$, exhibited the largest genetic load in both species. One possible explanation for this finding is that highly deleterious alleles might have been more efficiently purged from populations that went through more severe bottlenecks due to higher inbreeding [67]. For example, species whose populations underwent extreme bottlenecks show fewer mutations of high impact because extensive inbreeding makes highly deleterious alleles more likely to be exposed in homozygosity [104-106]. This is not the case for Downy and Hairy Woodpecker, which despite repeated episodes of bottlenecks still managed to maintain considerably large population sizes, making inbreeding very unlikely to have occurred. Besides, we found that Alaska, the population with the lowest long-term $\mathrm{N}_{\mathrm{e}}$, does not carry the fewest highly deleterious alleles, as predicted by the "purging under inbreeding" scenario. Instead, it carries a larger load than the East and the Pacific Northwest, which are populations with a higher long-term $\mathrm{N}_{\mathrm{e}}$. At the species level, however, we found that genetic load was generally larger in Downy Woodpecker than Hairy Woodpecker, which is consistent with more efficient purifying selection in Hairy Woodpecker. This finding makes sense considering that Hairy Woodpecker exhibits slightly larger $\mathrm{N}_{\mathrm{e}}$ than Downy Woodpecker. Supporting this observation, we also found a larger excess of highly deleterious mutations at low frequencies in Hairy Woodpecker, indicating that deleterious alleles were less likely to rise to high frequencies in Hairy Woodpecker than Downy Woodpecker likely due to more efficient selection. Lastly, we observed that the genome-wide ratio of non-synonymous over synonymous substitutions (dN/dS) was higher in Downy Woodpecker than Hairy Woodpecker. Elevated genome-wide, as opposed to genespecific, $\mathrm{dN} / \mathrm{dS}$ ratio is suggestive of a reduction in the efficacy of purifying selection [71,72]. This result indicates that a smaller $\mathrm{N}_{\mathrm{e}}$ in the lineage leading to Downy Woodpecker might have allowed more fixation of slightly deleterious alleles.

In conclusion, we investigated the impact of demography and natural selection on the genomic

517 landscape of two co-distributed woodpecker species whose population histories have been profoundly 518 impacted by the Ice Age. We found that despite a dynamic demographic history, Downy and Hairy 519 Woodpecker were able to maintain very large $\mathrm{N}_{\mathrm{e}}$ even during glacial periods, which might have facilitated 520 the action of natural selection. Supporting this conclusion, our results reveal a correlation between 521 nucleotide diversity, recombination rate, and gene density, which suggests the effect of linked selection 522 shaping the genomic landscape. In addition, we found that the magnitude of linked selection was associated 523 with population-specific $\mathrm{N}_{\mathrm{e}}$ trajectories, indicating that demography and natural selection operated in 524 concert to shape patterns of polymorphism along the genome. This study adds to the growing body of 525 literature supporting the role of natural selection in driving patterns of genome-wide variation but highlights 
526 the difficulty of interpreting the outcome of the interplay between genetic drift and natural selection in

527 organisms with non-equilibrium demographic dynamics and large effective population sizes.

\section{Material and Methods}

530 We collected 70 samples for both the Downy Woodpecker (D. pubescens) and Hairy Woodpecker (D.

531 villosus) in each of seven populations ( $n=10$ per population) across their temperate North American ranges

532 (Figure 1): New York (Northeast), Louisiana (Southeast), Minnesota (Midwest), New Mexico and

533 Colorado (Southern Rockies), Wyoming (Northern Rockies), Washington (Pacific Northwest), and Alaska.

534 The samples were obtained through museum loans of vouchered specimens and augmented by field

535 collections in Wyoming, Louisiana, and Alaska (Table S1). We extracted genomic DNA from tissue

536 samples using the MagAttract High Molecular Weight DNA Kit from Qiagen following manufacturer's

537 instructions (Qiagen, California, USA). These samples were then submitted for whole genome resequencing

538 on a paired-end Illumina HiSeq X Ten machine at RAPiD Genomics (Gainesville, Florida, USA).

\section{Read alignment, variant calling and filtering}

540 Raw reads were trimmed for Illumina adapters using Trimmomatic v0.36 [107] with the following 541 parameters: “ILLUMINACLIP:TruSeq3-PE-2.fa:2:30:10:8:true”, resulting in average of 35,689,979

542 paired reads per sample. Read quality was assessed with FastQC v0.11.4. [108]. Given the high synteny 543 and evolutionary stasis of bird chromosomes [82], we produced a chromosome-length reference genome 544 for Downy Woodpecker by ordering and orienting the scaffolds and contigs of the Downy Woodpecker 545 genome assembly [52] along the 35 chromosomes of the Zebra finch (Taeniopygia guttata; version 546 taeGut3.2.4) using Chromosemble from the Satsuma package [109]. We verified the completeness of this 547 new reference by searching for a set of single-copy avian orthologs using BUSCO v2.0.1 (Benchmarking 548 Universal Single-Copy Orthologs [110]). A total of $91.1 \%$ of these genes were present and complete in our 549 pseudo-chromosome reference, indicating sufficient completeness. We finally transferred the prediction550 based genome annotation of the Downy Woodpecker [52] by mapping the genomic coordinates of each 551 annotated feature against the pseudo-chromosome reference using gmap [111]. A total of $99.98 \%$ of all the

55214,443 annotated genes in Downy Woodpecker were successfully mapped to the pseudo-chromosome 553 reference.

554 Trimmed reads for both Downy and Hairy Woodpecker were aligned against the pseudo555 chromosome reference genome of the Downy Woodpecker using BWA v0.7.15 mem algorithm [112]. On 556 average, $97.27 \%$ of reads from Downy Woodpecker and $96.38 \%$ of reads from Hairy woodpecker were 
successfully mapped, demonstrating that despite the large evolutionary distance between these two species [47], sequence conservation allows efficient mapping. Resulting sequence alignment/map (SAM) files were converted to their binary format (BAM) and sequence group information was added. Next, reads were sorted, marked for duplicates, and indexed using Picard (http://broadinstitute.github.io/picard/). The Genome Analysis Toolkit (GATK v3.6 [113]) was then used to perform local realignment of reads near insertion and deletion (indels) polymorphisms. We first used the RealignerTargetCreator tool to identify regions where realignment was needed, then produced a new set of realigned binary sequence alignment/map (BAM) files using IndelRealigner. The final quality of mapping was assessed using QualiMap v.2.2.1 [114].

We implemented two complementary approaches for the downstream analysis of genetic polymorphism. First, we used ANGSD v0.917 [53], a method that accounts for the genotype uncertainty inherent to low depth sequencing data by inferring genotype likelihoods instead of relying on genotype calls. We estimated genotype likelihoods from BAM files using the GATK model (-GL 2 [113]), retaining only sites present in at least $70 \%$ of sampled individuals (-minInd 50) and with the following filters: a minimum mapping quality of 30 (-minMapQ 30), a minimum quality score of 20 (-minQ 20), a minimum frequency of the minor allele of 5\% (-minMaf 0.05), and a P-value threshold for the allele-frequency likelihood ratio test statistic of 0.01 (-SNP_pval 0.01). Allele frequencies were estimated directly from genotype likelihoods assuming known major and minor alleles (-doMajorMinor 1 -doMaf 1 ). A total of $16,736,465$ and 15,463,356 SNPs were identified for Downy and Hairy Woodpecker, respectively. Because several downstream analyses lack support for genotype likelihoods, we also called genotypes using GATK v3.8.0 [115]. First, we run HaplotypeCaller separately for each sample using the --emitRefConfidence GVCF -minPruning 1 -minDanglingBranchLength 1 options to create one gVCF per individual, then we ran GenotypeGVCFs with default settings across all samples to jointly call genotypes. In the absence of a training SNP panel for our non-model species, we applied hard filtering recommendations from the Broad Institute's Best Practices (https://gatk.broadinstitute.org/). We filtered SNPs with quality by depth below 2 $(\mathrm{QD}<2.0)$, SNPs where reads with the alternative allele were shorter than those with the reference allele (ReadPosRankSum < -8), SNPs with evidence of strand bias (FS > 60.0 and SOR > 3.0), SNPs with root mean square of the mapping quality below 40 (MQ < 40.0), and SNPs in reads where the alternative allele had a lower mapping quality than the reference allele (MQRankSumTest $<-12.5$ ). In addition, we used VCFtools v0.1.17 [116] to retain only biallelic SNPs occurring in at least $75 \%$ of samples, with a minimal mean coverage of $2 \mathrm{x}$, a maximum mean coverage of $100 \mathrm{x}$, and a P-value above 0.01 for the exact test for Hardy-Weinberg Equilibrium. We applied three different minor allele frequency (maf) thresholds -0.05

589 (for most analyses), 0.02 (for the estimation of recombination rates), and no threshold (for demographic 590 analyses based on the SFS). 
To assess population structure, we performed a principal components analysis (PCA) using the R package SNPRelate v3.3 [117]. We first applied the function snpgdsLDpruning to select a subset of unlinked SNPs ( $\mathrm{LD} \mathrm{r}^{2}$ threshold $=0.2$ ), with $<25 \%$ missing data and a maf $>0.05$, which resulted in a total of 71,228 SNPs for Downy Woodpecker and 71,763 SNPs for Hairy Woodpecker. We then used the function snpgdsPCA to calculate the eigenvectors and eigenvalues for the principal component analysis. We investigated population structure by looking at the first three principal components (PC1-PC3). In addition, we used NGSadmix [54], implemented in ANGSD [53], to investigate the number of genetic clusters, and associated admixture proportions for each individual. NGSadmix is a maximum likelihood approach analogous to STRUCTURE [118], but bases its inferences on genotype likelihoods instead of SNP calls, therefore accounting for the uncertainty of genotypes.

We also described the relationships among populations by building a maximum likelihood tree based on the polymorphism-aware phylogenetic model (PoMo [58]) implemented in IQ-Tree 2 [59]. PoMo is a phylogenetic method that accounts for incomplete lineage sorting inherent to population-level data by incorporating polymorphic states into DNA substitution models. We used a python script (https://github.com/pomo-dev/cflib) to convert our vcf files containing only intergenic SNPs into the input format of PoMo (counts file). IQ-Tree was run using the HKY+P model of sequence evolution with 100 non-parametric bootstraps to assess support. We used three samples from Hairy Woodpecker as an outgroup to root the tree for Downy Woodpecker, and vice versa.

We estimated pairwise $F_{\text {ST }}$ values among populations in each species using ANGSD v0.917 [53]. We first produced site-allele-frequency likelihoods using the command -doSaf, followed by the realSFS fold 1 command to generate a folded site frequency spectrum (SFS). We then estimated weighted $\mathrm{F}_{\mathrm{ST}}$ values using the realSFS fst command both globally and across non-overlapping $100 \mathrm{~kb}$ windows.

We investigated patterns of gene flow across the landscape using the estimated effective migration surface (EEMS [55]), which is a method to visualize variation in patterns of gene flow across a habitat.

616 Low values of relative effective migration rate $(m)$ indicate a rapid decay in genetic similarity in relation to

617 geographic distances, which suggests the presence of barriers to gene flow. In contract, high values of $m$ 618 indicate larger genetic similarity than expected given the geographic distance, suggesting genetic 619 connectivity. We generated pairwise identity-by-state (IBS) matrices using the -doIBS function in ANGSD 620 [53] and used these matrices to represent dissimilarity between individuals. We ran EEMS using 200 demes 621 and performed a single MCMC chain run with $1 \times 10^{7}$ iterations following a burn-in of $5 \times 10^{6}$, and a 622 thinning of 9,999. We then checked the posterior probabilities to ensure convergence. 
624 We inferred past changes in effective population size $\left(\mathrm{N}_{\mathrm{e}}\right)$ using Stairway Plot 2 [56], a method that

625 leverages information contained in the site frequency spectrum (SFS) to estimate recent population history.

626 Unlike methods based on the Sequentially Markov Coalescent (e.g, PSMC, SMC++), Stairway Plot 2 is

627 applicable to a large sample of unphased whole genome sequences, and it is insensitive to read depth

628 limitations. We estimated the folded site frequency spectrum for each population using the realSFS function

629 in ANGSD [53]. For each population, we used the default 67\% sites for training, and calculated median

630 estimates and 95\% pseudo-CI based on 200 replicates. We assumed a mutation rate of $4.007 \times 10^{-9}$

631 mutations per site per generation, as estimated from coding regions of the Northern Flicker's genome [57]

632 and a generation time of one year for both species. We then utilized the estimates of $\mathrm{N}_{\mathrm{e}}$ from Stairway Plot

6332 across the past 500 kya to calculate the harmonic mean on linear-stepped time points, representing each

634 population's long-term $\mathrm{N}_{\mathrm{e}}$.

We further investigated the demographic history of the two species using fastsimcoal2 v2.6.0.3, a composite likelihood method that uses the joint site frequency spectrum (jSFS) to perform model selection and estimate demographic parameters [62]. We tested the support for two competing demographic models: (i) a model where all populations diverge synchronously from a single large refugium and expand independently with asymmetric gene flow, and (ii) a bifurcating model where populations diverge at different times from multiple refugia and expand independently with asymmetric gene flow. Since we only need a reasonably large subset of the genome to get an accurate estimate of the site frequency spectrum

642 [119], we generated the four-population folded jSFS from a set of high quality SNPs with no maf filtering

643 (Downy: 6,030,759 SNPs; Hairy: 7,967,215 SNPs) present in chromosome 1 using easySFS.py

644 (https://github.com/isaacovercast/easySFS). We projected the jSFS down to 20 chromosomes (i.e., 10

645 diploid samples) per population to avoid issues associated with differences in sample size and missing data.

646 To minimize the impact of selection, we only included sites in non-coding regions of the genome. All

647 models followed the topology of the population tree obtained from IQ-Tree 2 and assumed a mutation rate

648 of $4.007 \times 10^{-9}$ mutations per site per generation. For each model, we conducted 75 iterations of the 649 optimization procedure, each with 40 expectation conditional maximization cycles and 100,000 650 genealogical simulations per cycle. We performed model selection using the run with the highest likelihood 651 for each model. For each species, we chose the model with the largest relative Akaike information criterion $652\left(\mathrm{AIC}_{\mathrm{w}}\right)$ as the best-fit model. We obtained $95 \%$ pseudo-CI for parameter estimates by performing 100 653 parametric bootstrap estimates simulating jSFSs under the best model and re-estimating parameters using 654 these simulated datasets.

655 Genetic diversity, recombination rates, and linkage disequilibrium 
656 We compared genetic diversity among populations of the two species by estimating the genome-wide

657 pairwise nucleotide diversity $\theta_{\pi}$ and the Watterson estimator of the rescaled mutation rate per base $\theta_{\mathrm{W}} \operatorname{sing}$

658 ANGSD [53]. We first ran the command -doSaf 1 -minMapQ 30 -minQ 20 in ANGSD to generate site-

659 allele-frequency likelihoods based on the GATK model [115], then we used -realSFS with the option -fold

6601 to estimate the folded SFS. ANGSD was also used to estimate genome-wide Tajima's D. We estimated

661 recombination rates ( $r=$ recombination rate per base pair per generation) along the genome of the two

662 species using ReLERNN, a deep learning algorithm [63]. ReLERNN takes as input a vcf file and simulates

663 training, validation, and test datasets matching the empirical distribution of $\theta_{\mathrm{w}}$. ReLERNN then uses the

664 raw genotype matrix and a vector of genomic coordinates to train a model that directly predicts per-base

665 recombination rates (as opposed to a population-scaled recombination rate) across sliding windows [63].

666 To reduce the impact of population structure on estimates, we restricted the prediction of recombination

667 rates to the Eastern populations (Northeast + Southeast + Midwest), the genetic cluster with most samples.

668 Given the conserved landscape of recombination in birds, we do not expect major differences in

669 recombination across populations [82]. We used the SNP dataset with maf $>0.02$ and ran the analysis with

670 default settings. Because ReLERNN is robust to demographic model misspecification [63], we simulated

671 an equilibrium model considering a mutation rate of $4.007 \times 10^{-9}$ mutations per generation [57] and

672 assuming a generation time of one year. Finally, we explored the recombination history of each population

673 by analyzing their patterns of linkage disequilibrium (LD) decay using PopLDdecay [120]. We calculated

674 pairwise $D^{\prime} / \mathrm{r}^{2}$ using the default maximum distance between SNPs of $300 \mathrm{~kb}$ and plotted it as a function of

675 genomic distance (in $\mathrm{kb})$.

Genomic predictors of regional variation in nucleotide diversity

677 To investigate the factors shaping the genomic landscape of diversity in the two woodpecker species, we

678 tested the effect of (i) recombination rate, (ii) gene density, and (iii) GC content on regional patterns of 679 nucleotide diversity. We computed pairwise nucleotide diversity $\left(\theta_{\pi}\right)$ across $100 \mathrm{~kb}$ non-overlapping 680 windows using ANGSD [53]. We first used the -doThetas function to estimate the site-specific nucleotide 681 diversity from the posterior probability of allele frequency (SAF) using the estimated site frequency 682 spectrum (SFS) as a prior. Then, we ran the thetaStat do_stat command to perform the sliding windows 683 analysis. To quantify variation in recombination rates, we calculated weighted averages of recombination 684 rates estimated in ReLERNN across $100 \mathrm{~kb}$ non-overlapping windows. We assessed gene density (i.e., 685 density of targets of selection) as the proportion of coding sequence (in number of base-pairs) for any given $686100 \mathrm{~kb}$ non-overlapping window and estimated GC content in each $100 \mathrm{~kb}$ non-overlapping window using 687 the function $G C$ of the R package seqinr version 3.6-1 [121]. We fit a general linear regression in $\mathrm{R}$ to 688 assess the relationship between nucleotide diversity $\left(\theta_{\pi}\right)$ and the three predictor variables - recombination 
rate, gene density, and base composition. We also fit a LOESS model to account for the potential nonlinearity of these relationships using the R package caret [122]. Models were trained using cross-validation of $80 \%$ of the total data. To control for the collinearity among these variables, we also ran a principal component regression (PCR). PCR is a technique that summarizes the predictor variables into orthogonal components (PCs) before performing regression, therefore removing the correlation among variables. PCR was conducted using the R package pls [123]. All variables were Z-transformed before these analyses.

We also investigated the association between patterns of intraspecific population differentiation

$\left(\mathrm{F}_{\mathrm{ST}}\right)$ and intrinsic properties of the genome (i.e., nucleotide diversity and recombination rates). To summarize the genomic landscape of differentiation into a single response variable we employed two approaches: for each $100 \mathrm{~kb}$ windows, we (i) calculated the average $\mathrm{F}_{\mathrm{ST}}$ across all pairwise population comparisons; (ii) we performed a principal component analysis and extracted that first principal component (PC1) that explained the greatest covariance among all pairwise population comparisons.

Natural selection and genetic load

To estimate the genetic load of each species and populations, we first used the software snpEff v4.1 [124] to classify SNPs into one of four categories of functional impact, according to the predicted effect of the gene annotation - (i) modifiers: variants in non-coding regions of the genome (e.g, introns, intergenic) whose effects are hard to predict; (ii) low: variants in coding sequences that cause no change in amino acid (i.e., synonymous); (iii) moderate: variants in coding sequences that cause a change in amino acid (i.e., nonsynonymous); and (iv) high: variants in coding sequences that cause gain or loss of start and stop codon. We then selected a subset of 12 individuals with the lowest percentage of missing data (therefore, maximizing the total number of sites) in each species to polarize our SNPs. To do so, we looked for biallelic SNPs in Downy Woodpecker for which one of the alleles were fixed in Hairy Woodpecker and vice versa.

711 The allele fixed in the outgroup was assumed to be the ancestral state. This is a sensitive step in the estimation of genetic load, so we only kept SNPs for which the ancestral state could be determined unambiguously [70]. We ended up with a total set of 363,903 polarized SNPs across the genome.

We characterized the site frequency spectrum (SFS) for each type of variant (according to the

715 impact inferred from snpEff) by estimating the total frequency of each derived allele and calculating the

716 proportion of each allele frequency bin. As a proxy for genetic load, for each individual, we estimated the

717 ratio of the number of derived alleles of high impact (i.e., loss of function) in homozygosity over the number

718 of derived alleles of low impact (i.e, synonymous) in homozygosity. This metric assumes a recessive model,

719 in which derived alleles are only deleterious when in a homozygous state. We therefore also considered an

720 additive model (i.e, semi-dominant) that assumes that derived alleles have deleterious effects in both 
homozygosity and heterozygosity. For this metric, we counted the total number of derived alleles, instead of only the ones in homozygosity [70].

To look at selection over a deeper evolutionary scale, we estimated $\mathrm{dN} / \mathrm{dS}$, the ratio of nonsynonymous over synonymous substitution, using a set of 397 genes that were orthologous across

725 Downy Woodpecker, Hairy Woodpecker and two avian outgroups - Chicken (Gallus gallus) and Zebra

726 Finch (Taeniopygia guttata). We identified orthologous genes across all four species using the software

727 JustOrthlogs [125] and only kept well-aligned loci. We first downloaded Ensembl genome assemblies and 728 gene annotations for version GRCg6a and bTaeGut1_v1.p of the Chicken and Zebra Finch genome, 729 respectively (Ensembl v103). We then extracted coding sequences (CDS) for all identified orthologs from 730 their respective reference genomes using a GFF3 parser included in JustOrthologs and aligned them with 731 the frameshift-aware MACSE software [126]. We used the parameter setting --min_percent_NT_at_ends 7320.3 and -codonForInternalStop $N N N$ for aligning and exporting sequences. The resulting amino-acid 733 alignments were inspected with HMMcleaner to mask sites that were likely misaligned. We finally used 734 codeml to estimate the overall $\mathrm{dN} / \mathrm{dS}$ ratio along each branch of the tree assuming a one-ratio branch model in PAML [127].

\section{Acknowledgments}

738 We thank the following individuals and institutions for providing tissue samples and specimen loans for 739 this study: A. G. Navarro Sigüenza (Museo de Zoología; UNAM), J. Klicka/S. Birk/R. Faucett (University 740 of Washington Burke Museum), C. M. Milensky (Smithsonian Institution), C. Dardia (Cornell University 741 Museum of Vertebrates), G. Spellman/A. Doll (Denver Museum of Nature \& Science), B. Marks/S. 742 Hackett/J. Bates (Field Museum of Natural History), F. Sheldon/D. Dittmann (LSU Museum of Natural 743 Science), K. Barker/T. Imfeld (Midwest Museum of Natural History), C. Witt/A. Johnson/M. Anderson 744 (Museum of Southwestern Biology). This work would not be possible without the assistance of Lucas 745 DeCicco, Matt Brady, and Paul Sweet, who were very generous to help collect samples in the field. We 746 thank Kaiya Provost, Jon Merwin, Vivien Chua, Glenn Seehozer, Gregory Thom, Elkin Tenorio, William 747 Mauck, Lukas Musher, Laís Coelho, Amanda Rocha, Bruno Almeida, Isaac Overcast, Joel Cracraft, Frank 748 Burbrink, Molly Przeworski, Deren Eaton, and Don Melnick for their invaluable input during the 749 development and writing of this manuscript.

750 Funding: This study was funded by the Department of Ecology, Evolution, and Environmental Biology 751 (E3B) at Columbia University, Conselho Nacional de Desenvolvimento Científico e Tecnológico (CNPq; 752 grant no. 211496/2014-6), the Frank M. Chapman Memorial Fund and Linda J. Gormezano Memorial Fund 753 from the American Museum of Natural History (AMNH), the American Ornithological Society Hesse 
Author contributions: This study was conceived and designed by L.R.M. and B.T.S. A subset of samples was collected and made available by J.K. L.R.M. conducted all wet lab, bioinformatic analyses, and drafted the paper with input from all authors.

Competing interests: The authors declare that they have no competing interests.

Data and materials availability: Whole-genome resequencing data are available at the Sequence Read Archive (https://ncbi.nlm.nih.gov/sra) under accession numbers \#. All data needed to evaluate the conclusions in the paper are present in the paper and/or the Supplementary Materials. Code used in this study is available at https://github.com/lucasrocmoreira/Moreira-et-al-2022. Additional data related to this paper may be requested from the authors.

\section{References}

766 1. Hewitt G. The genetic legacy of the Quaternary ice ages. Nature. 2000;405: 907-913.

2. Hewitt GM. Genetic consequences of climatic oscillations in the Quaternary. Willis KJ, Bennett KD, Walker D, editors. Philos Trans R Soc Lond B Biol Sci. 2004;359: 183-195.

3. Nadachowska-Brzyska K, Li C, Smeds L, Zhang G, Ellegren H. Temporal dynamics of avian populations during Pleistocene revealed by whole-genome sequences. Curr Biol. 2015;25: 13751380.

4. Lessa EP, Cook JA, Patton JL. Genetic footprints of demographic expansion in North America, but not Amazonia, during the Late Quaternary. Proceedings of the National Academy of Sciences. 2003;100: 10331-10334.

5. Burbrink F, Chan YL, Myers EA, Ruane S, Smith BT, Hickerson MJ. Asynchronous demographic responses to Pleistocene climate change in Eastern Nearctic vertebrates. Sgro C, editor. Ecol Lett. 2016;19: 1457-1467.

6. Knowles LL. Did the Pleistocene glaciations promote divergence? Tests of explicit refugial models in montane grasshopprers. Mol Ecol. 2001;10: 691-701.

7. Zink RM. The role of subspecies in obscuring avian biological diversity and misleading conservation policy. Proceedings of the Royal Society B: Biological Sciences. 2004;271: 561-564.

8. Anderson LL, Hu FS, Nelson DM, Petit RJ, Paige KN. Ice-age endurance: DNA evidence of a white spruce refugium in Alaska. Proceedings of the National Academy of Sciences. 2006;103: 1244712450. 
One. 2007;2: e563.

10. Shafer ABA, Cullingham CI, Côté SD, Coltman DW. Of glaciers and refugia: A decade of study sheds new light on the phylogeography of northwestern North America. Mol Ecol. 2010;19: 45894621.

11. Campbell-Staton SC, Goodman RM, Backström N, Edwards SV, Losos JB, Kolbe JJ. Out of Florida: mtDNA reveals patterns of migration and Pleistocene range expansion of the Green Anole lizard (Anolis carolinensis). Ecol Evol. 2012;2: 2274-2284.

12. Pulgarín-R PC, Burg TM. Genetic signals of demographic expansion in Downy Woodpecker (Picoides pubescens) after the Last North American Glacial Maximum. Johnson N, editor. PLoS One. 2012; 7: e40412.

13. Reid BN, Kass JM, Wollney S, Jensen EL, Russello MA, Viola EM, et al. Disentangling the genetic effects of refugial isolation and range expansion in a trans-continentally distributed species. Heredity . 2018. doi:10.1038/s41437-018-0135-5

14. Davis MB. Range shifts and adaptive responses to Quaternary climate change. Science. 2001;292: 673-679.

15. Gossmann TI, Shanmugasundram A, Börno S, Duvaux L, Lemaire C, Kuhl H, et al. Ice-Age Climate Adaptations Trap the Alpine Marmot in a State of Low Genetic Diversity. Curr Biol. 2019;29: 17121720.e7.

16. Li J, Li H, Jakobsson M, Li S, Sjödin P, Lascoux M. Joint analysis of demography and selection in population genetics: Where do we stand and where could we go? Mol Ecol. 2012;21: 28-44.

17. Kern AD, Hahn MW. The Neutral Theory in Light of Natural Selection. Kumar S, editor. Mol Biol Evol. 2018;35: 1366-1371.

18. Jensen JD, Payseur BA, Stephan W, Aquadro CF, Lynch M, Charlesworth D, et al. The importance of the Neutral Theory in 1968 and 50 years on: A response to Kern and Hahn 2018. Evolution. 2019;73: 111-114.

19. Kimura M, Crow JF. The number of alleles that can be maintained in a finite population. Genetics. 1964;49: 725-738.

20. Kimura M. The Neutral Theory of Molecular Evolution. Cambridge University Press; 1983.

21. Maynard J, Haigh J. The hitch-hiking effect of a favourable gene. Genet Res . 2007;89: 391-403.

22. Cutter AD, Choi JY. Natural selection shapes nucleotide polymorphism across the genome of the nematode Caenorhabditis briggsae. Genome Res. 2010;20: 1103-1111.

23. Charlesworth B, Morgan MT, Charlesworth D. The effect of deleterious mutations on neutral molecular variation. Genetics. 1993;134: 1289-1303.

24. Cutter AD, Payseur BA. Genomic signatures of selection at linked sites: unifying the disparity among species. Nat Rev Genet. 2013;14: 262-274.

25. Comeron JM. Background Selection as Baseline for Nucleotide Variation across the Drosophila Genome. Begun DJ, editor. PLoS Genet. 2014;10: e1004434. 
824

825

826

827

828

829

830

831

832

833

834

835

836

837

838

839

840

841

842

843

844

845

846

847

848

849

850

851

852

853

854

855

856

857

858

859

860

861

26. Henn BM, Botigué LR, Peischl S, Dupanloup I, Lipatov M, Maples BK, et al. Distance from subSaharan Africa predicts mutational load in diverse human genomes. Proc Natl Acad Sci U S A. 2016;113: E440-9.

27. Willi Y, Fracassetti M, Zoller S, Van Buskirk J. Accumulation of Mutational Load at the Edges of a Species Range. Mol Biol Evol. 2018;35: 781-791.

28. Wang XJ, Hu QJ, Guo XY, Wang K, Ru DF, German DA, et al. Demographic expansion and genetic load of the halophyte model plant Eutrema salsugineum. Mol Ecol. 2018;27: 2943-2955.

29. Rougemont Q, Moore J-S, Leroy T, Normandeau E, Rondeau EB, Withler RE, et al. Demographic history shaped geographical patterns of deleterious mutation load in a broadly distributed Pacific Salmon. Buerkle A, editor. PLoS Genet. 2020;16: e1008348.

30. de Pedro M, Riba M, González-Martínez SC, Seoane P, Bautista R, Claros MG, et al. Demography, genetic diversity and expansion load in the colonizing species Leontodon longirostris (Asteraceae) throughout its native range. Mol Ecol. 2021; mec.15802.

31. Mattila TM, Laenen B, Horvath R, Hämälä T, Savolainen O, Slotte T. Impact of demography on linked selection in two outcrossing Brassicaceae species. Ecol Evol. 2019;9: 9532-9545.

32. Begun DJ, Aquadro CF. Levels of naturally occurring DNA polymorphism correlate with recombination rates in D. melanogaster. Nature. 1992;356: 519-520.

33. Gossmann TI, Woolfit M, Eyre-Walker A. Quantifying the Variation in the Effective Population Size Within a Genome. Genetics. 2011;189: 1389-1402.

34. Dutoit L, Vijay N, Mugal CF, Bossu CM, Burri R, Wolf J, et al. Covariation in levels of nucleotide diversity in homologous regions of the avian genome long after completion of lineage sorting. Proceedings of the Royal Society B: Biological Sciences. 2017;284. doi:10.1098/rspb.2016.2756

35. Stankowski S, Chase MA, Fuiten AM, Rodrigues MF, Ralph PL, Streisfeld MA. Widespread selection and gene flow shape the genomic landscape during a radiation of monkeyflowers. Jiggins CD, editor. PLoS Biol. 2019;17: e3000391.

36. Wang J, Street NR, Park E, Liu J, Ingvarsson PK. Evidence for widespread selection in shaping the genomic landscape during speciation of Populus. Mol Ecol. 2020;29: 1120-1136.

37. Talla V, Soler L, Kawakami T, Dincă V, Vila R, Friberg M, et al. Dissecting the Effects of Selection and Mutation on Genetic Diversity in Three Wood White (Leptidea) Butterfly Species. Gonzalez J, editor. Genome Biol Evol. 2019;11: 2875-2886.

38. Andolfatto P. Hitchhiking effects of recurrent beneficial amino acid substitutions in the Drosophila melanogaster genome. Genome Res. 2007;17: 1755-1762.

39. Branca A, Paape TD, Zhou P, Briskine R, Farmer AD, Mudge J, et al. Whole-genome nucleotide diversity, recombination, and linkage disequilibrium in the model legume Medicago truncatula. Proceedings of the National Academy of Sciences. 2011;108: E864-E870.

40. Beissinger TM, Wang L, Crosby K, Durvasula A, Hufford MB, Ross-Ibarra J. Recent demography drives changes in linked selection across the maize genome. Nat Plants. 2016;2: 16084.

41. Jensen-Seaman MI. Comparative Recombination Rates in the Rat, Mouse, and Human Genomes. 
Genome Res. 2004;14: 528-538.

863

864

865

866

867

868

869

870

871

872

873

874

875

876

877

878

879

880

881

882

883

884

885

886

887

888

889

890

891

892

893

894

895

896

897

898

42. Smukowski CS, Noor MAF. Recombination rate variation in closely related species. Heredity . 2011;107: 496-508.

43. Kawakami T, Smeds L, Backström N, Husby A, Qvarnström A, Mugal CF, et al. A high-density linkage map enables a second-generation collared flycatcher genome assembly and reveals the patterns of avian recombination rate variation and chromosomal evolution. Mol Ecol. 2014;23: 4035-4058.

44. Schield DR, Pasquesi GIM, Perry BW, Adams RH, Nikolakis ZL, Westfall AK, et al. Snake recombination landscapes are concentrated in functional regions despite PRDM9. Mol Biol Evol. 2020;37: 1272-1294.

45. Ouellet HR. Biosystematics and ecology of Picoides villosus (L.) and P. pubescens (L.) (Aves Picidae). McGill University. 1977.

46. Weibel AC, Moore WS. Plumage convergence in Picoides woodpeckers based on a molecular phylogeny, with emphasis on convergence in Downy and Hairy woodpeckers. Condor. 2005;107: 797-809.

47. Dufort MJ. An augmented supermatrix phylogeny of the avian family Picidae reveals uncertainty deep in the family tree. Mol Phylogenet Evol. 2016;94: 313-326.

48. Klicka J, Spellman GM, Winker K, Chua V, Smith BT. A phylogeographic and population genetic analysis of a widespread, sedentary North American bird: The Hairy Woodpecker (Picoides villosus). Auk. 2011;128: 346-362.

49. Graham BA, Burg TM. Molecular markers provide insights into contemporary and historic gene flow for a non-migratory species. J Avian Biol. 2012;43: 198-214.

50. Avise J. Mitochondrial DNA Phylogeographic Differentiation among Avian Populations and the Evolutionary Significance of Subspecies. Auk. 1992;109: 626-636.

51. Smith BT, Gehara M, Harvey MG. The demography of extinction in eastern North American birds. Proceedings of the Royal Society B: Biological Sciences. 2021;288: 20201945.

52. Jarvis ED, Mirarab S, Aberer AJ, Li BB, Houde P, Li C, et al. Whole-genome analyses resolve early branches in the tree of life of modern birds. Science. 2014;346: 1320-1331.

53. Korneliussen TS, Albrechtsen A, Nielsen R. ANGSD: Analysis of Next Generation Sequencing Data. BMC Bioinformatics. 2014;15: 356.

54. Skotte L, Korneliussen TS, Albrechtsen A. Estimating individual admixture proportions from next generation sequencing data. Genetics. 2013;195: 693-702.

55. Petkova D, Novembre J, Stephens M. Visualizing spatial population structure with estimated effective migration surfaces. Nat Genet. 2016;48: 94-100.

56. Liu X, Fu Y-X. Stairway Plot 2: demographic history inference with folded SNP frequency spectra. Genome Biol. 2020;21: 280.

57. Hruska JP, Manthey JD. De novo assembly of a chromosome-scale reference genome for the 
northern flicker Colaptes auratus. G3 . 2021;11. doi:10.1093/g3journal/jkaa026

900

901

902

903

904

905

906

907

908

909

910

911

912

913

914

915

916

917

918

919

920

921

922

923

924

925

926

927

928

929

930

931

932

933

934

935

58. Schrempf D, Minh BQ, De Maio N, von Haeseler A, Kosiol C. Reversible polymorphism-aware phylogenetic models and their application to tree inference. J Theor Biol. 2016;407: 362-370.

59. Minh BQ, Schmidt HA, Chernomor O, Schrempf D, Woodhams MD, von Haeseler A, et al. IQTREE 2: New Models and Efficient Methods for Phylogenetic Inference in the Genomic Era. Mol Biol Evol. 2020;37: 1530-1534.

60. Pruett CL, Winker K. Evidence for cryptic northern refugia among high- and temperate-latitude species in Beringia. Clim Change. 2008;86: 23-27.

61. Brubaker LB, Anderson PM, Edwards ME, Lozhkin AV. Beringia as a glacial refugium for boreal trees and shrubs: new perspectives from mapped pollen data. J Biogeogr. 2005;32: 833-848.

62. Excoffier L, Foll M. fastsimcoal: a continuous-time coalescent simulator of genomic diversity under arbitrarily complex evolutionary scenarios. Bioinformatics. 2011;27: 1332-1334.

63. Adrion JR, Galloway JG, Kern AD. Predicting the landscape of recombination using deep learning. Wilke C, editor. Mol Biol Evol. 2020; 1-27.

64. Sundström H, Webster MT, Ellegren H. Reduced Variation on the Chicken Z Chromosome. Genetics. 2004. pp. 377-385. doi:10.1534/genetics.167.1.377

65. Xu L, Wa Sin SY, Grayson P, Edwards SV, Sackton TB. Evolutionary Dynamics of Sex Chromosomes of Paleognathous Birds. Genome Biol Evol. 2019;11: 2376-2390.

66. Zhou Q, Zhang J, Bachtrog D, An N, Huang Q, Jarvis ED, et al. Complex evolutionary trajectories of sex chromosomes across bird taxa. Science. 2014;346: 1246338-1246338.

67. Kirkpatrick M, Jarne P. The Effects of a Bottleneck on Inbreeding Depression and the Genetic Load. Am Nat. 2000;155: 154-167.

68. Charlesworth B. Fundamental concepts in genetics: effective population size and patterns of molecular evolution and variation. Nat Rev Genet. 2009;10: 195-205.

69. Simons YB, Turchin MC, Pritchard JK, Sella G. The deleterious mutation load is insensitive to recent population history. Nat Genet. 2014;46: 220-224.

70. Simons YB, Sella G. The impact of recent population history on the deleterious mutation load in humans and close evolutionary relatives. Curr Opin Genet Dev. 2016;41: 150-158.

71. Elyashiv E, Bullaughey K, Sattath S, Rinott Y, Przeworski M, Sella G. Shifts in the intensity of purifying selection: An analysis of genome-wide polymorphism data from two closely related yeast species. Genome Res. 2010;20: 1558-1573.

72. Figuet E, Nabholz B, Bonneau M, Mas Carrio E, Nadachowska-Brzyska K, Ellegren H, et al. Life History Traits, Protein Evolution, and the Nearly Neutral Theory in Amniotes. Mol Biol Evol. 2016;33: 1517-1527.

73. Herrera-Álvarez S, Karlsson E, Ryder OA, Lindblad-Toh K, Crawford AJ. How to Make a Rodent Giant: Genomic Basis and Tradeoffs of Gigantism in the Capybara, the World's Largest Rodent. Rogers R, editor. Mol Biol Evol. 2020. doi:10.1093/molbev/msaa285 
74. Renaut S, Grassa CJ, Yeaman S, Moyers BT, Lai Z, Kane NC, et al. Genomic islands of divergence are not affected by geography of speciation in sunflowers. Nat Commun. 2013;4: 1827.

75. Burri R, Nater A, Kawakami T, Mugal CF, Olason PI, Smeds L, et al. Linked selection and recombination rate variation drive the evolution of the genomic landscape of differentiation across the speciation continuum of Ficedula flycatchers. Genome Res. 2015;25: 1656-1665.

76. Van Doren BM, Campagna L, Helm B, Illera JC, Lovette IJ, Liedvogel M. Correlated patterns of

77. Delmore KE, Lugo Ramos JS, Van Doren BM, Lundberg M, Bensch S, Irwin DE, et al. Comparative analysis examining patterns of genomic differentiation across multiple episodes of population divergence in birds. Evolution Letters. 2018;2: 76-87.

78. Dutoit L, Burri R, Nater A, Mugal CF, Ellegren H. Genomic distribution and estimation of nucleotide diversity in natural populations: perspectives from the collared flycatcher ( Ficedula albicollis ) genome. Mol Ecol Resour. 2017;17: 586-597.

79. Ellegren H. Evolutionary stasis: the stable chromosomes of birds. Trends Ecol Evol. 2010;25: 283291.

80. Volker M, Backstrom N, Skinner BM, Langley EJ, Bunzey SK, Ellegren H, et al. Copy number variation, chromosome rearrangement, and their association with recombination during avian evolution. Genome Res. 2010;20: 503-511.

81. Ellegren H. The evolutionary genomics of birds. Annu Rev Ecol Evol Syst. 2013;44: 239-259.

82. Singhal S, Leffler EM, Sannareddy K, Turner I, Venn O, Hooper DM, et al. Stable recombination hotspots in birds. Science. 2015;350: 928-932.

83. Reich DE, Cargill M, Bolk S, Ireland J, Sabeti PC, Richter DJ, et al. Linkage disequilibrium in the human genome. Nature. 2001;411: 199-204.

84. Ardlie KG, Kruglyak L, Seielstad M. Patterns of linkage disequilibrium in the human genome. Nat Rev Genet. 2002;3: 299-309.

85. Balakrishnan CN, Edwards SV. Nucleotide variation, linkage disequilibrium and founder-facilitated speciation in wild populations of the zebra finch (Taeniopygia guttata). Genetics. 2009;181: 645660.

86. Kardos M, Husby A, McFarlane SE, Qvarnström A, Ellegren H. Whole-genome resequencing of extreme phenotypes in collared flycatchers highlights the difficulty of detecting quantitative trait loci in natural populations. Mol Ecol Resour. 2016;16: 727-741.

87. Wilson Sayres MA. Genetic diversity on the sex chromosomes. Genome Biol Evol. 2018;10: 10641078.

88. Irwin DE. Sex chromosomes and speciation in birds and other ZW systems. Mol Ecol. 2018;27: 3831-3851.

89. Levin I, Crittenden LB, Dodgson JB. Genetic Map of the Chicken Z Chromosome Using Random Amplified Polymorphic DNA (RAPD) Markers. Genomics. 1993;16: 224-230. 
90. Schmid M, Nanda I, Guttenbach M, Steinlein C, Hoehn M, Schartl M, et al. First report on chicken genes and chromosomes 2000. Cytogenet Genome Res. 2000;90: 169-218.

91. Borge T, Webster MT, Andersson G, Saetre G-P. Contrasting Patterns of Polymorphism and Divergence on the Z Chromosome and Autosomes in Two Ficedula Flycatcher Species. Genetics. 2005;171: 1861-1873.

92. Mank JE, Axelsson E, Ellegren H. Fast-X on the Z: rapid evolution of sex-linked genes in birds.

93. Mugal CF, Nabholz B, Ellegren H. Genome-wide analysis in chicken reveals that local levels of

94. Wang J, Street NR, Scofield DG, Ingvarsson PK. Natural selection and recombination rate variation shape nucleotide polymorphism across the genomes of three related populus species. Genetics. 2016;202: 1185-1200.

95. Corbett-Detig RB, Hartl DL, Sackton TB. Natural selection constrains neutral diversity across a wide range of species. Barton NH, editor. PLoS Biol. 2015;13: e1002112.

96. Cruickshank TE, Hahn MW. Reanalysis suggests that genomic islands of speciation are due to reduced diversity, not reduced gene flow. Mol Ecol. 2014;23: 3133-3157.

97. Matthey-Doret R, Whitlock MC. Background selection and F ST : Consequences for detecting local adaptation. Mol Ecol. 2019;28: 3902-3914.

98. Vijay N, Weissensteiner M, Burri R, Kawakami T, Ellegren H, Wolf JBW. Genomewide patterns of variation in genetic diversity are shared among populations, species and higher-order taxa. Mol Ecol. 2017;26: 4284-4295.

99. Irwin DE, Alcaide M, Delmore KE, Irwin JH, Owens GL. Recurrent selection explains parallel evolution of genomic regions of high relative but low absolute differentiation in a ring species. Mol Ecol. 2016;25: 4488-4507.

100. Jackson LE Jr. New evidence for the existence of an icefree corridor in the Rocky Mountain foothills near Calgary, Alberta, during Late Wisconsinan time. Anat Physiol. 1979; 107-111.

101. Rutter NW. Pleistocene history of the western Canadian ice-free corridor. Quaternary stratigraphy of Canada: a Canadian contribution to the IGCP Project. 1984;24: 49-56.

102. Pedersen MW, Ruter A, Schweger C, Friebe H, Staff RA, Kjeldsen KK, et al. Postglacial viability and colonization in North America's ice-free corridor. Nature. 2016; 1-15.

103. Ohta T. Slightly deleterious mutant substitutions in evolution. Nature. 1973;246: 96-98.

104. Xue Y, Prado-Martinez J, Sudmant PH, Narasimhan V, Ayub Q, Szpak M, et al. Mountain gorilla genomes reveal the impact of long-term population decline and inbreeding. Science. 2015;348: 242245. 
106. Grossen C, Guillaume F, Keller LF, Croll D. Purging of highly deleterious mutations through severe bottlenecks in Alpine ibex. Nat Commun. 2020;11: 1001.

107. Bolger AM, Lohse M, Usadel B. Trimmomatic: a flexible trimmer for Illumina sequence data. Bioinformatics. 2014;30: 2114-2120.

108. Andrews S. FastQC: a quality control tool for high throughput sequence data. Babraham Bioinformatics, Babraham Institute, Cambridge, United Kingdom; 2010.

109. Grabherr MG, Russell P, Meyer M, Mauceli E, Alföldi J, Di Palma F, et al. Genome-wide synteny through highly sensitive sequence alignment: Satsuma. Bioinformatics. 2010;26: 11451151.

110. Waterhouse RM, Seppey M, Simão FA, Manni M, Ioannidis P, Klioutchnikov G, et al. BUSCO applications from quality assessments to gene prediction and phylogenomics. Mol Biol Evol. 2018;35: 543-548.

111. Wu TD, Watanabe CK. GMAP: a genomic mapping and alignment program for mRNA and EST sequences. Bioinformatics. 2005;21: 1859-1875.

112. Li H, Durbin R. Fast and accurate short read alignment with Burrows-Wheeler transform. Bioinformatics. 2009;25: 1754-1760.

113. DePristo MA, Banks E, Poplin R, Garimella KV, Maguire JR, Hartl C, et al. A framework for variation discovery and genotyping using next-generation DNA sequencing data. Nat Genet. 2011;43: 491-498.

114. Okonechnikov K, Conesa A, García-Alcalde F. Qualimap 2: advanced multi-sample quality control for high-throughput sequencing data. Bioinformatics. 2016;32: 292-294.

115. McKenna A, Hanna M, Banks E, Sivachenko A, Cibulskis K, Kernytsky A, et al. The Genome Analysis Toolkit: A MapReduce framework for analyzing next-generation DNA sequencing data. Genome Res. 2010;20: 1297-1303.

116. Danecek P, Auton A, Abecasis G, Albers CA, Banks E, DePristo MA, et al. The variant call format and VCFtools. Bioinformatics. 2011;27: 2156-2158.

117. Zheng X, Levine D, Shen J, Gogarten SM, Laurie C, Weir BS. A high-performance computing toolset for relatedness and principal component analysis of SNP data. Bioinformatics. 2012;28: 3326-3328.

118. Pritchard JK, Stephens M, Donnelly P. Inference of population structure using multilocus genotype data. Genetics. 2000;155: 945-959.

119. Beichman AC, Huerta-Sanchez E, Lohmueller KE. Using Genomic Data to Infer Historic Population Dynamics of Nonmodel Organisms. Annu Rev Ecol Evol Syst. 2018 [cited 8 Feb 2021]. doi:10.1146/annurev-ecolsys-110617-062431

120. Zhang C, Dong S-S, Xu J-Y, He W-M, Yang T-L. PopLDdecay: a fast and effective tool for linkage disequilibrium decay analysis based on variant call format files. Schwartz R, editor. Bioinformatics. 2018; 1-3.

1047 121. Charif D, Lobry JR. SeqinR 1.0-2: A Contributed Package to the R Project for Statistical 
1048

Computing Devoted to Biological Sequences Retrieval and Analysis. In: Bastolla U, Porto M, Roman HE, Vendruscolo M, editors. Structural Approaches to Sequence Evolution: Molecules, Networks, Populations. Berlin, Heidelberg: Springer Berlin Heidelberg; 2007. pp. 207-232.

122. Kuhn M, Others. Building predictive models in R using the caret package. J Stat Softw. 2008;28: $1-26$.

123. Wehrens R, Mevik B-H. The pls package: principal component and partial least squares regression in R. 2007. Available: https://repository.ubn.ru.nl/bitstream/handle/2066/36604/36604.pdf

124. Cingolani P, Platts A, Wang LL, Coon M, Nguyen T, Wang L, et al. A program for annotating and predicting the effects of single nucleotide polymorphisms, SnpEff. Fly . 2012;6: 80-92.

125. Miller JB, Pickett BD, Ridge PG. JustOrthologs: a fast, accurate and user-friendly ortholog identification algorithm. Bioinformatics. 2019;35: 546-552.

126. Ranwez V, Harispe S, Delsuc F, Douzery EJP. MACSE: Multiple Alignment of Coding SEquences Accounting for Frameshifts and Stop Codons. PLoS ONE. 2011. p. e22594. doi:10.1371/journal.pone.0022594

127. Yang Z. PAML 4: phylogenetic analysis by maximum likelihood. Mol Biol Evol. 2007;24: 15861591. 